Aus der Klinik für Psychiatrie und Psychotherapie (Prof. Dr. med. J. Wiltfang)

der Medizinischen Fakultät der Universität Göttingen

\title{
Decis State - Dysfunktionen neuronaler Mechanismen der Impulskontrolle bei Suchterkrankung - eine funktionell-kernspintomographische Untersuchung von Patienten mit langjähriger Alkoholabhängigkeit
}

\author{
INAUGURAL - DISSERTATION \\ zur Erlangung des Doktorgrades \\ der Medizinischen Fakultät der \\ Georg-August-Universität zu Göttingen
}

vorgelegt von

Jasmin Said, geb. Turkani

aus

Herat/ Afghanistan

Göttingen 2019 
Dekan:

Referent:

Ko-Referentin:

Drittreferent/in:
Prof. Dr. rer. nat. H. K. Kroemer

i.V. Prof. Dr. Dirk Wedekind

Prof. Dr. Melanie Wilke

Prof. Dr. Margarete Schön

Datum der mündlichen Prüfung: 27.05.2020 
Hiermit erkläre ich, die Dissertation mit dem Titel „Decis State Dysfunktionen neuronaler Mechanismen der Impulskontrolle bei Suchterkrankung eine funktionell-kernspintomographische Untersuchung von Patienten mit langjähriger Alkoholabhängigkeit" eigenständig angefertigt keine anderen als die von mir angegebenen Quellen und Hilfsmittel verwendet zu haben.

Göttingen, den 31.Januar 2019 


\section{INHALTSVERZEICHNIS}

\section{Abkürzungsverzeichnis}

1 EINLEITUNG

1.1 Klinische Grundlagen des Alkoholismus ..................................................1

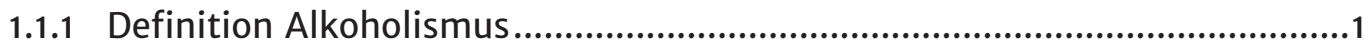

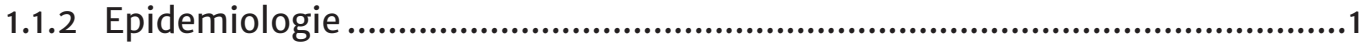

1.1.3 Pathophysiologie der Abhängigkeit .............................................................. 2

1.1.4 Alkoholbedingte Folgeschäden ................................................................. 3

1.2 Relevante neurofunktionelle Systeme ......................................................... 4

1.2.1 Das Dopaminerge Belohnungssystem ......................................................... 4

1.2.2 Motivation und Impulskontrolle ................................................................... 5

1.2.3 Studienergebnisse bei Alkoholabhängigkeit.................................................. 6

1.3 Ziele der Studie ............................................................................................... 7

2 MATERIAL UND METHODEN 10

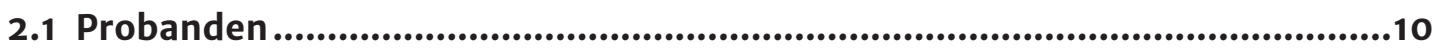

2.1.1 Ausschluss von Probanden......................................................................... 10

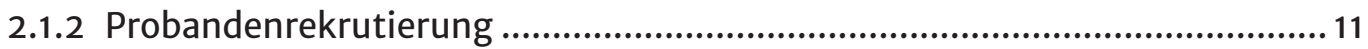

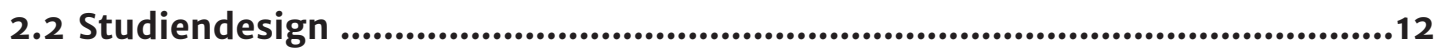

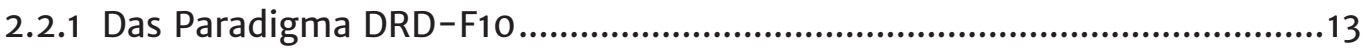

2.2.1.1 Stimulusmaterial ......................................................................................... 15

2.2.1.2 Versuchsablauf .......................................................................................... 17

2.3 Geräte und Messinstrumente ......................................................................18

2.3.1 Demographie und Fragebögen .................................................................... 18

2.3.1.1 Allgemeiner Fragebogen ..........................................................................18

2.3.1.2 Visuelle Analogskala für Substanz-Craving (VAS) ...................................... 18

2.3.1.3 Fragebogen zum Trinkverhalten ..............................................................18

2.3.2 Funktionelle Bildgebung............................................................................. 19

2.3.3 Statistische Analyse der Verhaltensdaten ...................................................20

2.3.4 Statistische Analyse der fMRT-Daten ......................................................20 
3.1 Fragebögen................................................................................................ 23

3.1.1 Visuelle Analogskala für Substanz-Craving (VAS) ..................................... 23

3.1.2 Fragebogen zum Trinkverhalten ...................................................................24

3.2 Verhaltensdaten......................................................................................... 25

3.3 Funktionelle Bildgebung .............................................................................. 27

3.3.1 Unterschiede bei konditionierten nicht alkoholischen Stimuli................... 27

3.3.2 Unterschiede bei alkoholischen Stimuli........................................................ 30

3.3.3 Top-Down-Suppression in Desire-Reason-Dilemma-Situation und bei Ablehnung von alkoholischen Stimuli .............................................................31

1 DISKUSSION 33

5 AUSBLICK $\quad 40$

6 ZUSAMMENFASSUNG

$\begin{array}{lll}7 \text { ANHANG } & 43\end{array}$

7.1 Tabellen ................................................................................................................. 43

7.2 Abbildungen ..................................................................................................... 46

7.3 Stimulusmaterial............................................................................................ 48

7.4 Fragebögen............................................................................................... 50

7.5 Kontrastliste .................................................................................................... 55

8 LITERATURVERZEICHNIS $\quad 56$ 


\section{ABKÜRZUNGSVERZEICHNIS}

ACC anteriorer zingulärer Kortex

Alk alkoholisches Getränk

ALKNT alkoholisches Non-Target

ANOVA analysis of variance

ANT alkoholisches Non-Target

avPFC anteroventraler präfrontaler Kortex

dIPFC dorsolateraler präfrontaler Kortex

BOLD blood oxygen level dependency

DRD Desire-Reason-Dilemma

DSM Diagnostic and Statistical Manual of Mental Disorders

EPI echo planar imaging

fMRT funktionelle Magnetresonanztomographie

GABA $\quad \gamma$-Aminobuttersäure

GLM general linear model

HPC Hippocampus

HRF hemodynamic response function

ICD International Statistical Classification of Diseases

KNT konditioniertes Non-Target

MNI Montreal Neurological Institute

NAc Nucleus Accumbens

NBS Neurobehavioralsystem

NMDA N-Methyl-D-Aspartat

Non-Alk nicht-alkoholisches Getränk

NT Non-Target

OBJ Objekt

OFC orbitofrontaler Kortex

PET Positronen-Emissions-Tomographie

PFC präfrontaler Kortex 
pg ACC prägenuale ACC

sg ACC subgenuale ACC

SPM Statistical Parametric Mapping

T Target

TE time echo

TR time repetition

vm PFC ventromedialer präfrontaler Kortex

VSt ventrales Striatum

VTA ventrales tegmentales Areal

WHO World Health Organization 


\section{EINLEITUNG}

\subsection{Klinische Grundlagen des Alkoholismus}

\subsubsection{Definition Alkoholismus}

Der Terminus Alkoholismus wurde 1852 von dem schwedischen Arzt Huss geprägt und umfasst zwei Phänomene: Alkoholabhängigkeit und Alkoholmissbrauch. Kennzeichen von Alkoholabhängigkeit sind nach Definition der WHO: ein starker Wunsch bzw. Zwang, Alkohol zu konsumieren, der Kontrollverlust über Beginn, Beendigung und Menge des Alkoholkonsums, Toleranzentwicklung, Entzugserscheinungen, Vernachlässigung von anderen Interessen und Vergnügen zugunsten des Alkoholkonsums und letztlich anhaltender Konsum trotz des Wissens über die schädlichen Folgen (DSM IV 2003; WHO 2008). Dagegen liegt nach Definition der WHO Alkoholmissbrauch vor, wenn der Konsum von Alkohol zu körperlichen-, psychischen- oder sozialen Folgeschäden führt, jedoch noch keine Alkoholabhängigkeit vorliegt (DSM IV 2003; WHO 2008).

\subsubsection{Epidemiologie}

Die Suchterkrankungen gehören mittlerweile zu einem der größten Problemgebiete des heutigen Gesundheitswesens weltweit. Nach Tabak belegt Alkohol den zweiten Platz der am meisten missbrauchten Substanzen mit Schwerpunkt in den Industrienationen. Danach folgen suchtpotente Medikamente und illegale Rauschmittel. Alle Daten zur Epidemiologie des Alkoholkonsums in Deutschland beruhen auf den Selbstangaben von Befragten. Laut einem Bericht des Robert KochInstituts aus dem Jahr 2002 trinken 30\% der Männer mehr als 20g Alkohol pro Tag und 16\% der Frauen täglich mehr als $10 \mathrm{~g}$ (Bühringer 2000; Burger et al. 2003). Der höchste Konsum tritt im Alter von ca. 50 Jahren 
auf, wobei Raucher mehr trinken und in der oberen Sozialschicht ebenfalls mehr konsumiert wird (Bergmann und Horch 2002). Das führt zu einer erhöhten Morbiditäts - und Mortalitätsrate. Alleine in Deutschland sterben jährlich 40.000 Menschen an den Folgen schädlichen Alkoholkonsums (DHS 2017).

\subsubsection{Pathophysiologie der Abhängigkeit}

Die Suchterkrankung ist eine chronisch rezidivierende Rückfallerkrankung, die gekennzeichnet ist durch (1) zwanghaftes Verhalten, das Suchtmittel aufzusuchen und zu konsumieren, (2) Kontrollverlust bei Einschränkung des Konsums und (3) das Auftreten von negativen emotionalen Zuständen, z. B. Dysphorie, Angstzustände und Reizbarkeit. Besteht der Zugang zum Suchtmittel nicht, spiegeln letztere ein motivationales Entzugssyndrom wider (Koob und Le Moal 1997). Die Erkrankung weist Aspekte sowohl von Impulskontrollstörungen als auch von Zwangsstörungen auf, deren Zusammenspiel den sogenannten Suchtzyklus ergibt (American Psychiatric Association 1998). Dieser besteht aus drei Phasen: Intoxikation, Entzug und Verlangen (Craving). Als neuronale Kernregionen dieses Zyklus wurden unter

anderem das ventrale Tegmentum (VTA), das ventrale Striatum (VSt), die Amygdala, der Hippocampus (HPC), der orbitofrontale Kortex (OFC), der Gyrus cinguli und die Insula identifiziert (American Psychiatric Association 1998). Im frühen Stadium dominiert die mit positiver Verstärkung assoziierte Impulsivität, die sich später in Richtung Zwanghaftigkeit verschieben kann. Die Zwanghaftigkeit ist wiederum mit negativer Verstärkung assoziiert (Koob 2004). Diese Stadien interagieren intensiv miteinander und führen schließlich zu einem pathologischen Zustand, der als Sucht bekannt ist (Koob und Le Moal 1997). Nach einer Schätzung von Koob und Kollegen ist 40-60\% der Suchtgefährdung auf genetische Faktoren zurückzuführen. Die positiv verstärkende bzw. belohnende Wirkung von suchtpotenten Drogen 
während des Rausches setzt den Anfang, welche bei der Fortführung eines Missbrauchs zu neuronalen Anpassungen und schließlich zur Abhängigkeit führen kann. Mindestens vier neurofunktionelle Systeme spielen hierbei eine wichtige Rolle: das mesolimbische Dopaminsystem, das Opioidpeptidsystem, das $\gamma$-Aminobuttersäuresystem und das Endocannabinoidsystem einschließlich des Nucleus Accumbens (NAc) und der Amygdala (Koob 2008).

Alkohol führt über die neuronalen Anpassungen zur Toleranzentwicklung (Goldstein und Volkow 2002). Durch die Stimulation der GABA-Rezeptoren und die Hemmung der NMDA-Rezeptoren wirkt der Alkohol entspannend und angstlösend. Allerdings führt die Dauerstimulation des GABA-System zu der obengenannten Toleranzentwicklung (Schäfer und Heinz 2005). Es muss letztendlich immer mehr Alkohol zugeführt werden, um die gleichen Wirkung zu erzielen. Außerdem zeigen weitere Studien, dass eine subtile kognitive und emotionale Instabilität der Individuen zur Entstehung, Eskalation und Aufrechthaltung des Suchtzyklus beiträgt.

\subsubsection{Alkoholbedingte Folgeschäden}

Alkohol trägt mit großer Wahrscheinlichkeit zur Entstehung von zahlreichen körperlichen und seelischen Erkrankungen bei. Hierbei spielt die genetisch bedingte individuelle Verträglichkeit des Alkohols, die Toleranzentwicklung und die unterschiedliche Empfindlichkeit der einzelnen Organsysteme gegenüber der toxischen Wirkung des Alkohols eine zentrale Rolle. Neben der akuten Alkoholintoxikation und dem Alkohol-Entzugssyndrom zählen Erkrankungen der Leber, der Bauchspeicheldrüse, des Magen-Darm-Trakts, des Herzkreislaufsystems und des Bewegungsapparats zu den wichtigsten physischen Folgeerkrankungen.

$\mathrm{Zu}$ den neurologisch und psychiatrisch schweren Folgeschäden zählen vor allem die schwere Hirnatrophie, das Wernicke-Korsakow- 
Syndrom,Alkoholdelir, Alkohol-Polyneuropathie, depressiveEpisoden, schwere Angstzustände, Schlafstörungen, eine erhöhte Suizidrate und der Missbrauch von suchtpotenten Medikamenten. Vor allem aber auch in ihrem sozialen Umfeld sind die Betroffenen schwer beeinträchtigt (Bergmann und Horch 2002; Feuerlein 2005).

Volkswirtschaftlich verursacht Alkoholismus enorme Kosten. Nach Berechnungen von Adams und Effertz betrugen sie für Deutschland im Jahr 2007 insgesamt 26,7 Mrd. Euro (Adams und Effertz 2011; DHS 2017).

\subsection{Relevante neurofunktionelle Systeme}

\subsubsection{Das Dopaminerge Belohnungssystem}

Zur Deckung der Bedürfnisse einer Spezies wie beispielsweise Essen, Schlafen oder Reproduktion hat sich im Laufe der Evolution das sogenannte Belohnungssystem entwickelt. Entdeckt wurde dies zufällig durch die US-Forscher J. Old und P. Miller an Laborratten. Sie fanden heraus, dass die elektrische Stimulation mittels eingepflanzter Elektroden im Bereich des NAc und des anterioren zingulären Kortex (ACC) einen angenehmen Effekt erzeugte und zur Verstärkung des Verhaltens führte (Olds und Milner 1954).

Der sogenannte kortikale Basalganglien-Kreislauf spielt eine zentrale Rolle im Belohnungssystem. Nach Haber und Knutson zählen zu den Schlüsselstrukturen in diesem Netzwerk der ACC, der Präfrontalkortex (PFC) im Orbitalbereich, das VSt, das ventrale Pallidum und die Dopamin-Neuronen des Mittelhirns. Darüber hinaus sind anderen Strukturen, wie der dorsale präfrontale Kortex ( $\mathrm{dPFC}$ ), die Amygdala, der HPC, der Thalamus, die laterale Habenula und weitere spezifische Hirnstammstrukturen Schlüsselkomponenten für die Regulierung des Belohnungskreislaufs. Diese Strukturen bilden ein komplexes neuronales Netzwerk, das die verschiedenen Aspekte der Belohnungsverarbeitung 
regelt (Haber und Knutson 2010).

Hirnbildgebende Untersuchungen bei Menschen haben gezeigt, dass die dopaminergen Areale, insbesondere NAc, bei schnellen (impulsiven) Entscheidungen für präferierte bzw. hedone (attraktive) oder konditionierte Stimuli eine zentrale Rolle spielen (Havemann und Kuschinsky 1985; Yacubian et al. 2007) und ganz besonders auf Belohnung ausgerichtet sind (Walter et al. 2005). Präfrontale Areale, insbesondere der PFC repräsentieren eine „kontrollierte“ Form der Handlungskontrolle, im Rahmen derer Informationen über sowohl unmittelbare als auch in der Zukunft zu erwartende Handlungskonsequenzen integriert werden. Sie erlauben impulsive Handlungstendenzen zu unterdrücken, falls diese mit langfristigen Nachteilen verbunden sind (Daw et al. 2005).

\subsubsection{Motivation und Impulskontrolle}

Laut Berridge und Robinson beinhaltet Belohnung drei Komponenten: Motivation, Lernen und Affekte/Emotionen, die durch ihre verschiedenen impliziten und expliziten psychologischen Aspekte miteinander interagieren (Berridge und Robinson 2003). Die incentive-sensitization-theory of addiction von Berridge und Robinson geht von einer Sensitiverung mesolimbischer Areale durch suchtpotente Substanzen aus. Diese neuroadaptiven Prozesse führen dazu, dass die suchtmittelassoziierten Stimuli einen höheren Anreizwert (Salienz) besitzen und sich dieser Anreizwert im Verlauf der Suchtentwicklung steigert (sensitization of incentive salience) und so ein pathologisches Verlangen nach Suchtmittelkonsum induziert. Der hedonische Wert bleibt jedoch konstant oder nimmt sogar ab. Bereits kleine Mengen eines Suchtmittels können starke motivationale Reaktionen bewirken(Robinson und Berridge 1993).

Das menschliche Verhalten ist darauf ausgerichtet, Belohnung zu maximieren, jedoch negative Konsequenzen zu minimieren (Williams 
2006). Zu diesem Zweck braucht es eine adaptive Handlungskontrolle, im Rahmen derer speziell auch langfristige Handlungskonsequenzen berücksichtigt werden, um ggf. unmittelbare Handlungstendenzen zu unterdrücken, sofern diese langfristig negative Folgen haben. Die notwendigen Mechanismen haben sich im Laufe der Evolution entwickelt, um in entscheidenden Situationen diese präpotenten, impulsiven Handlungstendenzen kontrollieren zu können (Kalenscher et al. 2006). Diekhof und Gruber konnten zeigen, dass die Überwindung unmittelbar präferierter Handlungsimpulse mit dem Ziel, auf lange Sicht Belohnung zu maximieren, mit einer verstärkten negativen Kopplung des anteroventralen präfrontalen Kortex (avPFC) und des NAc einhergeht. In Situationen, in denen ein präferierter Handlungsimpuls überwunden werden musste, um langfristige Belohnungsmaximierung zu erreichen, war zudem die Aktivität im NAc signifikant reduziert, was darauf hinweist, dass der avPFC striatale Belohnungssignale moduliert und inhibiert (Diekhof und Gruber 2010). Letztlich basieren zielgerichtete Entscheidungen auf einem gemeinsamen Wertesignal, welches im ventromedialen präfrontalen Kortex (VmPFC) kodiert ist und die Modulation dieses Wertes durch den dorsolateralen präfrontalen Kortex (dlPFC) trägt zur Selbstkontrolle bei (Hare et al. 2009).

\subsubsection{Studienergebnisse bei Alkoholabhängigkeit}

Alkohol und andere suchtpotente Drogen stimulieren die Dopamin-Freisetzung im VSt, welche wiederum eine Substanzaufnahme verstärkt. In einer fMRT-Studie bei entgifteten Alkoholikern haben Heinz und Kollegen gezeigt, dass alkoholassoziierte Stimuli bei Alkoholikern im Vergleich zu gesunden Probanden einen höheren Anreizwert (Salienz) besaßen und zu Suchtdruck bzw. übermäßiger Aktivierung der neuronalen Netzwerke führten (Heinz et al. 2004).

In den letzten Jahren spielte in der Erforschung der Pathogenese von Suchterkrankungen und die Untersuchung von Funktionen bzw. 
Dysfunktionen neurofunktioneller Systeme eine zunehmend große Rolle. Der Fokus lag hierbei insbesondere auf den kognitiven und affektiven Funktionen, die bei Suchtpatienten nachgewiesenermaßen gestört sind. Neben Defiziten in der motivationalen Verarbeitung und der Fähigkeit Verhaltensimpulse zu unterdrücken, sind bei Suchtpatienten Veränderungen in der Funktion und Struktur präfrontaler Kortizes, insbesondere orbitofrontaler und frontomedialer Kortex, sowie dopaminerger mesolimbischer Areale z. B. NAc, von Bedeutung (Havemann und Kuschinsky 1982; Kalivas und Volkow 2005; Diekhof et al. 2008). So führt eine Desinhibition mesolimbischer Areale, z. B. nach einer Läsion der präfrontalen Areale, zu einer Verringerung der Impulskontrolle (Bechara et al. 1999). Ursächlich hierfür könnte unter anderem eine Verringerung bzw. Störung präfrontaler Einflüsse auf das VSt bzw. den NAc sein. Bislang ist noch wenig über die tatsächlichen Beziehungen zwischen den an Entstehung und Aufrechthaltung der Sucht beteiligten präfronto-mesolimbischen Netzwerken bekannt.

\subsection{Ziele der Studie}

Wie oben beschrieben, gehen Suchterkrankungen mit Veränderungen in Hirnregionen einher, die an Emotions- und Belohnungsverarbeitung bzw. ihrer Kontrolle beteiligt sind. In humanen fMRT-Studien wurden durch Belohnungsreize verursachte Aktivierungen im NAc (O'Doherty 2004; Williams 2006; Robinson und Berridge 1993) und in der VTA nachgewiesen (Adcock et al. 2006). Zielgerichtete Entscheidungen basieren auf einem gemeinsamen Wertesignal, das im vmPFC kodiert ist, und moduliert durch den dlPFC zur Selbstkontrolle beiträgt (Hare et al. 2009). Die Fähigkeit, unmittelbaren Belohnungen zu widerstehen, ist für den langfristigen Erfolg und das individuelle Wohlbefinden entscheidend. Inhibierende Einflüsse des avPFC auf das mesolimbische Dopaminsystem ermöglichen die Kontrolle über impulsive Entscheidungen bei Verlangen 
nach unmittelbaren Belohnungen (Diekhof et al. 2012a). Die Vorstudien aus der Arbeitsgruppe basierten auf dem sogenannten DRD-Paradigma (Desire-Reason-Dilemma) und ermöglichten eine Untersuchung des dopaminergen Belohnungssystems samt den Hirnstrukturen, die an Beurteilungs- und Entscheidungsprozessen beteiligt sind. Sie zeigten bei gesunden Probanden einen inhibierenden Einfluss des PFC auf das Striatum, speziell bei der Ablehnung von belohnenden Ereignissen zugunsten eines langfristigen übergeordneten Zieles (Diekhof und Gruber 2010). Andere Studien mit entgifteten Alkoholikern fanden einen höheren Anreizwert (Salienz) alkoholassoziierter Stimuli. Im Vergleich zu gesunden Probanden führten sie zu Suchtdruck und übermäßiger Aktivierung des medialen PFC und Striatum (Heinz et al. 2004).

Das Ziel dieser Studie war es, die oben genannten Dysfunktionen dieser spezifischen neurofunktionellen Systeme bei Patienten mit langjähriger Alkoholsucht zu untersuchen. Das Hauptinteresse galt der Modulation von Belohnungssignalen in striatalen Regionen durch den PFC bei Veränderungen der neuronalen Mechanismen der Impulskontrolle. Um dieses im MRT untersuchen zu können, wurde ausgehend vom DRD-Paradigma von der Arbeitsgruppe das DRD-F10-Paradigma entwickelt. Als Stimulusmaterial wurden Bilder von alkoholischen und nicht-alkoholischen Getränken bzw. Objekten oder Sachen des täglichen Bedarfs verwendet, die im Rahmen der Diplomarbeit von Andrea Sauer in der Arbeitsgruppe entwickelt und validiert wurden (Sauer 2014). Die experimentelle Situation hatte große Ähnlichkeit mit realen Situationen im Leben von Suchtpatienten, in denen der kurzfristigen Versuchung, das Suchtmittel zu konsumieren widerstanden werden muss, um langfristig die Lebensqualität aufrechtzuerhalten.

Basierend auf den oben genannten Befunde lassen sich folgende Hypothesen definieren: Konditionierte Belohnungsreize sollten in beiden Gruppen zu einer erhöhten Aktivierung des extended reward system führen. Wie beim DRD-Paradigma müssten die Belohnungsareale des 
mesolimbischen Dopaminsystems und die integrierenden präfrontalen Areale zuverlässig aktiviert werden (Diekhof und Gruber 2010; Diekhof et al. 2012a). Die Anwendung des DRD-F10-Paradigmas sollte helfen, die Dysfunktion spezifischer neurofunktioneller Systeme bei der Suchterkrankung zu untersuchen. Als Reaktion auf alkoholische Stimuli wurde eine erhöhte Aktivierung der Belohnungsareale erwartet. $\mathrm{Zu}$ diesem Zweck wurden entgiftete Alkoholsuchtpatienten und gesunde Kontrollen einer fMRT-Untersuchung unterzogen, während sie das DRD-F10-Paradigma bearbeiteten.

\section{Zusammenfassung der Ziele:}

(H1) Wir erwarteten eine erhöhte Aktivierung des Belohnungssystems auf konditionierte Belohnungsreize.

(H2) In der Alkoholsuchtgruppe erwarteten wir eine erhöhte Aktivierung der Belohnungsareale insbesondere bei alkoholischen Stimuli.

(H3) Beim Vergleich der neuronalen Aktivitäten in einer Desire-ReasonDilemma-Situation, während Ablehnung von konditionierten Stimuli, erwarteten wir einen Suppressionseffekt in besagten Hirnarealen. 


\section{MATERIAL UND METHODEN}

\subsection{Probanden}

Im Rahmen der Studie wurden 17 Patienten mit der Diagnose Alkoholabhängigkeit ICD 10 F10.2, mindestens 14 Tage nach der letzten Entzugsbehandlung und 15 gesunde Kontrollprobanden untersucht. Davon 12 weibliche und 20 männliche Probanden im Alter von 18 bis 60 Jahren (Patienten: $M W=51,82, S D=4,343$, Kontrollen: $M W=26,13$, $\mathrm{SD}=7,282)$. Die personenbezogenen und psychometrischen Daten wurden mittels einer Probanden-Nummer pseudonymisiert ausgewertet und archiviert. Die Studie wurde von der Ethikkommission der Georg-August-Universität Göttingen genehmigt unter der Antragsnummer 13/5/11. Die Einwilligung zur freiwilligen Teilnahme war zur jederzeit widerrufbar.

\subsubsection{Ausschluss von Probanden}

Von den insgesamt 37 teilnehmenden Probanden wurden 3 Patienten und 2 Kontrollen aufgrund von Abbrüchen oder Bewegungsartefakten aus der Studie herausgenommen.

Tabelle 1: Demographische Daten, Ein- und Ausschluss von Probanden

\begin{tabular}{|l|c|c|c|}
\hline \multirow{2}{*}{ Geschlecht } & Kontrollgruppe & Patientengruppe \\
\cline { 2 - 4 } & W & 8 & 12 \\
\hline Alter & MW & 7 & 5 \\
\hline Eingeschlossen & 17 & $51,82($ SD 7,282) \\
\hline Ausgeschlossen & 3 & 15 \\
\hline Gesamt & 20 & 17 \\
\hline
\end{tabular}




\subsubsection{Probandenrekrutierung}

Die abstinenten Patienten mit gesicherter ICD 10 F10.2-Diagnose wurden nach Absprache mit dem Ärzteteam aus der Klinik für Psychiatrie und Psychotherapie der Universitätsmedizin Göttingen und durch die Versuchsleiterin während dem stationären-, oder dem Tagesklinikaufenthalt bzw. per Telefon und Email rekrutiert. Die gesunden Kontrollen wurden hauptsächlich über Aushänge an der Georg-August-Universität Göttingen und in Göttingen rekrutiert. Es galten folgende Ein- und Ausschlusskriterien für Patienten und Kontrollen:

\section{Einschlusskriterien}

- Alter zwischen 18 und 60 Jahren

- Diagnose der Alkoholabhängigkeit nach ICD 10 F10.2, frühestens 14 Tage nach der letzten Entzugsbehandlung

- Einwilligungsfähigkeit ist vorhanden

\section{Ausschlusskriterien}

- illegaler Drogenkonsum (einschließlich Cannabis) im letzten Monat

- relevante somatische Beschwerden, die nach Art und Schwere mit den geplanten Untersuchungen interferieren, Einfluss auf die zu untersuchenden Parameter haben oder den Probanden gefährden könnten (vor allem bekannte zerebrale Raumforderung, zerebrale degenerative bzw. entzündliche Erkrankungen, Herzkreislauferkrankungen, Herz-Schrittmacher, Metallimplantate, Schilddrüsenerkrankungen, Visusminderung, Epilepsie, Glaukom, BPH oder schwere auch ZNS-wirksame Stoffwechselerkrankungen)

- schwerwiegende psychiatrische Erkrankungen mit psychotischer oder dementiver Symptomatik

- psychiatrische Vor- oder bestehende Medikation außer Escitalopram, 
Citalopram oder Mirtazapin

- Schwangerschaft, Stillzeit oder positiver Schwangerschaftstest

- nicht vorhandene Lese-/Schreibfähigkeit

- akute Entzugssymptomatik, welche eine erfolgreiche Bearbeitung der experimentellen Aufgaben (verschiedene Gedächtnis- und Entscheidungsaufgaben) prinzipiell ausschließt

- akute Suizidalität

- Klaustrophobie

- nicht vorhandene Aufklärungs- oder Einwilligungsfähigkeit

- bei Kontrollen die Diagnose ICD 10 F 10.2 oder andere psychische Störungen

Patienten, die sich auf der Grundlage einer Unterbringung nach PsychKG oder einer gerichtlichen Betreuung in Behandlung befanden, wurden nicht in die Studie eingeschlossen.

Bei den Patienten erfolgte im Anschluss an die Untersuchung eine außerordentliche therapeutische Sitzung, in deren Fokus die Verarbeitung der Untersuchungssituation und speziell - falls gegeben - eine Auslösung von Suchtdruck durch die im MR verwendeten Stimuli im Fokus stand.

\subsection{Studiendesign}

Ziel der Studie war es, mittels funktioneller Magnetresonanztomographie potentielle krankheitsbezogene Veränderungen neuronaler Mechanismen der Impulskontrolle und der Belohnungsverarbeitung bei Alkoholabhängigkeit zu untersuchen. In diesem Zusammenhang interessierten insbesondere funktionelle Aktivierungen zerebraler Strukturen im avPFC einerseits und des NAc anderseits, wie sie in früheren Studien mit Impulskontrolle in Verbindung gebracht wurden (Diekhof und Gruber 2010). 


\subsubsection{Das Paradigma DRD-F10}

Die Untersuchung im MRT erfolgte mit dem in der Arbeitsgruppe entwickelten DRD-F10-Paradigma. Es ist eine modifizierte Version des DRD-Paradigmas, das Rückschlüsse auf belohnungsassoziierte Hirnaktivierungen und deren Interaktion zulässt, während konditionierte Stimuli zugunsten eines langfristigen Ziels angenommen oder nicht angenommen wurden (Diekhof et al. 2012a). Bei dem DRD-Paradigma handelt es sich um ein durch vorausgehende Studien etabliertes Verfahren zur Untersuchung des dopaminergen Belohnungssystems und der an Beurteilungs- und Entscheidungsprozessen beteiligten Hirnareale (Diekhof und Gruber 2010; Diekhof et al. 2012a).

Am ersten Tag vor dem Experiment wurden zwei Bilder aus der Kategorie nicht-alkoholische Getränke operant konditioniert. Hierbei wurden am Computer mit dem Programm Presentation (Version 14,4, Neurobehavioralsystems, Inc., CA, USA) alle Bilder einzeln und nacheinander präsentiert. Der Proband war dazu aufgefordert mit der linken Pfeiltaste auf jedes Bild zu reagieren. Nach dem Tastendruck erschien auf jedem Bild ein Feedback. Die zwei konditionierten Bilder wurden mit 10 Punkten belohnt. Alle anderen Bilder wurden nicht belohnt.

Am Tag des Experiments im MRT wurde zunächst die eigentliche Aufgabe trainiert.

Die Probanden mussten ein übergeordnetes langfristiges Ziel verfolgen, das am Ende eines jeden Aufgabenblocks mit 50 Punkten belohnt wurde (siehe Abbildung 1). Zu Beginn eines einzelnen Blocks wurde ein Hinweisreizbild (Cue) aus der Kategorie der nicht-alkoholischen (Non-alk) Bilder gezeigt. Dieses Bild galt während des aktuellen Blocks als zielführendes Bild [Target $(\mathrm{T})$ ]. Anschließend wurden jeweils zwei Bilder aus allen Kategorien gezeigt: Neutrales Bild Non-Target (NT), konditioniertes Bild (KNT) alkoholisches Bild (ALKNT) oder neutrales Objektbild (OBJ) (s. Anhang). Wenn eines der beiden Bilder identisch 
mit dem Hinweisreizbild bzw. Target T war, dann musste es ausgewählt werden, um das langfristige Ziel zu erreichen. In allen anderen Situationen war die Wahl frei. Wenn eines der Bilder den konditionierten Bildern, KNT, entsprach, so erhielten die Probanden 10 zusätzliche Punkte, wenn sie es auswählten. Hierbei entstand in DesireSituation die positive Verstärkung des Belohnungssystems durch die konditionierten 10 Punkte. Wenn das Hinweisreizbild gleichzeitig mit einem konditionierten Bild KNT präsentiert wurde, entstand eine Dilemma-Situation, da die konditionierten Bilder zugunsten eines langfristigen Ziels abgelehnt wurden. Hierbei mussten die Probanden immer das Hinweisreizbild T auswählen, da sonst das Aufgabenziel (50 Punkte) nicht erreicht wurde. Die zusätzlichen Bonuspunkte wurden am Ende jedes erfolgreichen Blocks zu den 50 Punkten addiert. Zu Beginn des Trainings und der Messung im Scanner wurden die Probanden darauf hingewiesen, dass sie alle nicht konditionierten neutralen, NT-, ALKNT- oder OBJ-Bilder nach ihren persönlichen Präferenzen auswählen sollten. Besonders wurde den Patienten erläutert, dass sie auch die alkoholischen Bilder ALKNT auswählen dürfen. Sie wurden über den Sinn und Zweck der Studie aufgeklärt und über die neuen wissenschaftlichen Erkenntnisse, die durch das Experiment erwartet wurden, informiert. Sie wurden ausdrücklich darauf hingewiesen, dass sie nicht in irgendeiner Art und Weise kontrolliert werden sollen. Die Präsentationsdauer betrugen für das Hinweisreizbild $1200 \mathrm{~ms}$, für die Trials jeweils 2200 ms und für das Block-Feedback 3000 ms. 


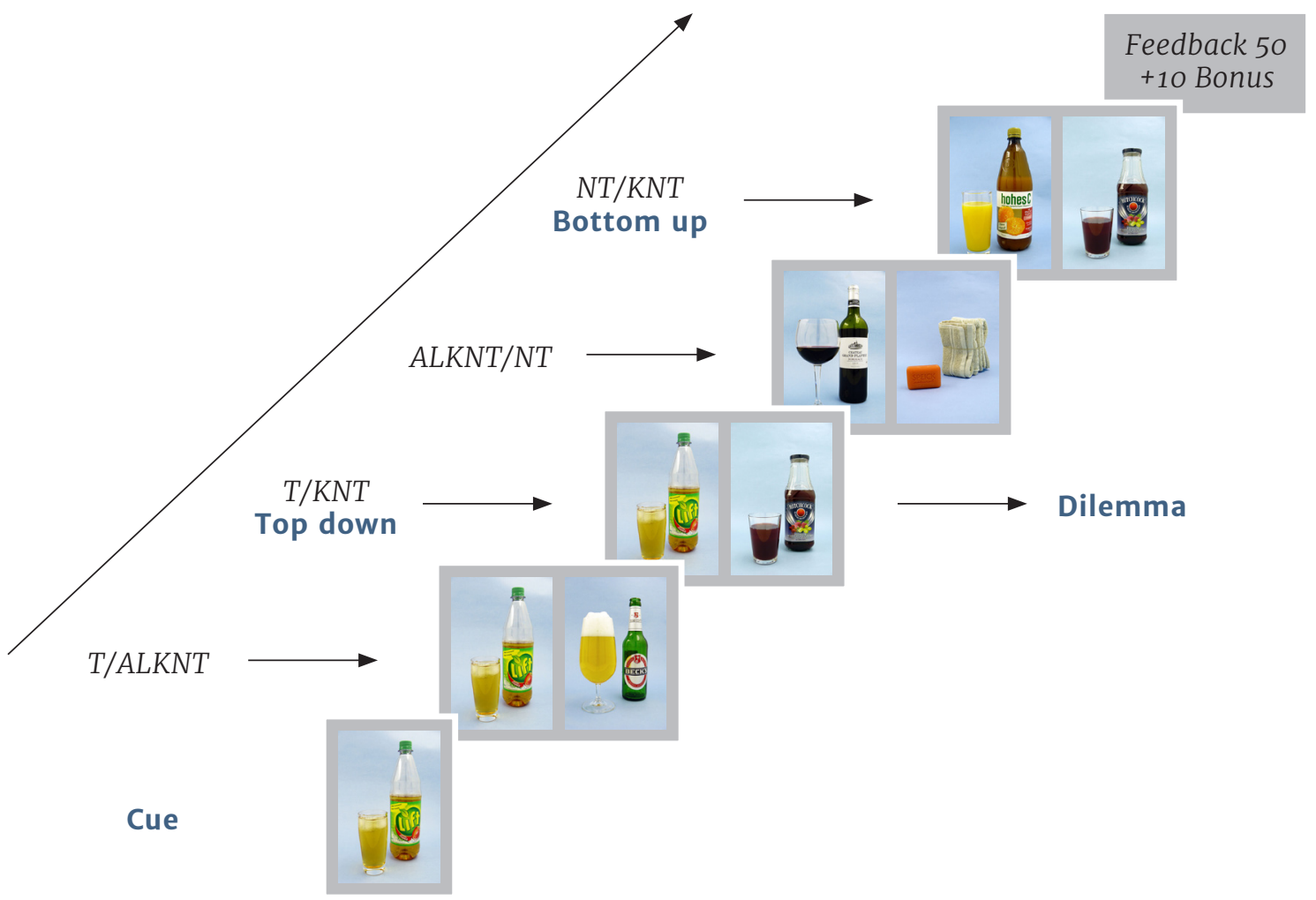

Abbildung 1: DRD-F1o-Paradigma: Die Probanden führten ein Entscheidungsfindungsparadigma aus, wobei sie sich zwischen unmittelbarer und langfristiger Belohnung entscheiden mussten. Das übergeordnete Ziel am Ende jedes erfolgreich bearbeiteten Blocks waren 50 Punkte. Am Anfang eines einzelnen Blocks wurde ein Hinweisreizbild (Cue) gezeigt, welches als Target (T) zielführend innerhalb jenes Blocks ausgewählt werden musste. Um zusätzliche 10 Bonuspunkte zu erhalten, konnten auch zuvor konditionierte Bilder (KNT) akzeptiert werden, wenn diese nicht parallel mit T präsentiert wurden (Dilemma). Eine fehlerhafte Annahme eines KNT bei paralleler Präsentation mit einem T führte zum Verlust von 50 Punkten.

Als Aufwandsentschädigung erhielten die Probanden $30 €$ und abhängig von ihrer Leistung einen prozentualen Bonus von maximal $10 €$, wenn alle Bonuspunkte erreicht wurden. Im Durchschnitt erhielten sie einen Bonus von $9 €$ (insgesamt $39 €)$.

\subsubsection{Stimulusmaterial}

Für die Studie wurden jeweils 16 Bilder aus den Kategorien alkoholische (Alk)-, nichtalkoholische Getränke (Non-alk) und neutrale 
Objekte (OBJ; Objekte des täglichen Bedarfs) ausgewählt (s. Anhang). Die Stimuli wurden in der Arbeitsgruppe von Prof. Dr. Oliver Gruber im Rahmen der Diplomarbeit von Andrea Sauer über die „Erstellung und Validierung eines Bildinventars zur Untersuchung krankheitsbezogener motivationaler Prozesse bei Alkoholabhängigkeit", speziell für das DRD-Paradigma F10 entwickelt. In dieser Arbeit wurde bei abstinenten Alkoholpatienten und Kontrollen die autonome physiologische Reaktion (Hautleitfähigkeitsreaktion) gemessen, während die oben genannten Stimuli auf einem Bildschirm präsentiert wurden. Außerdem wurde das subjektive Erleben der Probanden mittels graphisch unterstützter Ratingskalen erfasst. Es wurde eine erhöhte Hautleitfähigkeitsreaktion der Alkoholpatienten auf alkoholische Stimuli beobachtet (Sauer 2014).

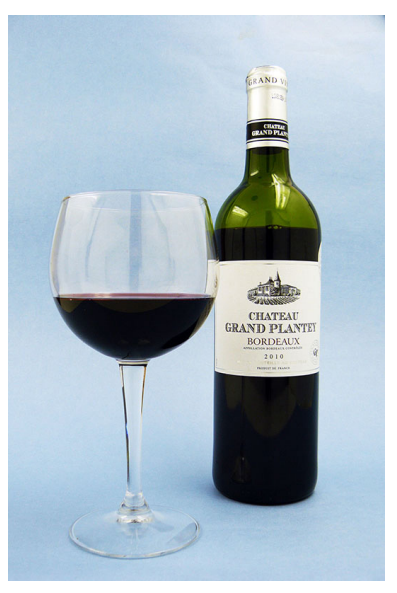

a) Alk

= alkoholisches Getränk

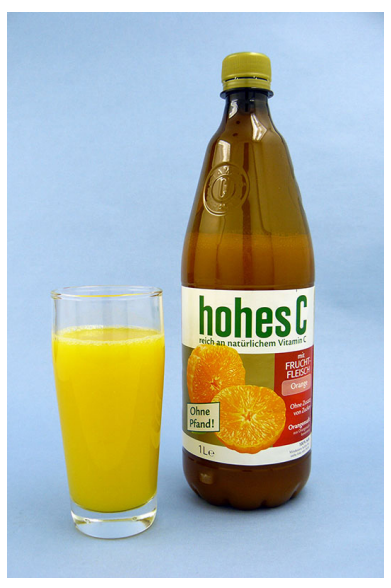

a) Non-alk

= nicht-alkoholisches Getränk

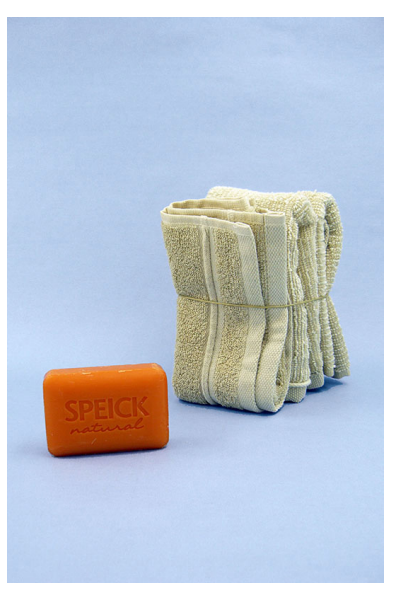

a) $\mathrm{OBJ}$

$=$ neutrales Objekt

Abbildung 2: Beispielbilder 


\subsubsection{Versuchsablauf}

Das Experiment erfolgte an zwei aufeinander folgenden Tagen. Der erste Teil fand im Testlabor der Abteilung für systemischen Neurowissenschaften der Universitätsmedizin Göttingen statt. Zunächst erfolgte ein Aufklärungsgespräch, im Rahmen dessen die Probanden über das Ziel und den Ablauf der Studie informiert wurden. Weiterhin wurden die Ein- und Ausschlusskriterien sowie die Eignung für die MR-Untersuchung überprüft. Anschließend unterschieben die Probanden die Einverständniserklärung für die fMRT-Untersuchung, einen Probandeninformationsbogen über die Studie und einen soziodemographischen Bogen.

Im Anschluss erfolgte, nach einer mündlichen und schriftlichen Instruktion, die Konditionierung am Computer mit der Software „Presentation". Nach einer mündlichen Überprüfung, ob der Proband sich die relevanten Stimuli richtig eingeprägt hatte, war die Untersuchung beendet. Der Zeitaufwand für den ersten Tag betrug ca. 30 Minuten.

Der zweite Teil des Experiments beinhaltete das Training der eigentlichen Aufgabe und die fMRT-Messung in den Räumen des Forschungs-MRT der Universitätsmedizin Göttingen (Leiter PD Dr. Dechent). Zunächst erfolgte nach der mündlichen und schriftlichen Instruktion und der Beantwortung von eventuell entstandenen Verständnisfragen, das Training des Experiments am Laptop. Nach einer kurzen Pause füllte der Proband die erste visuelle Analogskala (VAS) vor der MR-Messung aus und anschließend erfolgte die Untersuchung im MRT. Im Anschluss daran füllte der Proband noch einmal die VAS und den Fragebogen zum Trinkverhalten aus. Die Gesamtdauer für den zweiten Tag betrug ca. 1,5 Stunden. 


\subsection{Geräte und Messinstrumente}

\subsubsection{Demographie und Fragebögen}

\subsubsection{Allgemeiner Fragebogen}

In einem allgemeinen Fragebogen wurden Alter, Geschlecht, Größe und Gewicht der Probanden dokumentiert. Auch die wichtigsten Einund Ausschlusskriterien wurden schriftlich dokumentiert. Alle Fragebögen wurden im Kapitel Anhang aufgeführt.

\subsubsection{Visuelle Analogskala für Substanz-Craving (VAS)}

Die VAS für Substanz-Craving ist eine subjektive Eischätzungsskala, die auf der Grundlage von Measurement of Pain (Huskisson 1974), einer visuellen Analogskala für Schmerz, entwickelt wurde (Wedekind et al. 2010). Angewendet wurde die VAS jeweils unmittelbar vor und nach der MR-Messung. Die Skala enthielt zwei Fragen, anhand derer die Probanden auf einer Skala von o bis 100 a) ihren aktuellen Suchtdruck einschätzen sollten und b) wie gut sie diesen kontrollieren konnten. Die Auswertung erfolgte mittels SPSS.

\subsubsection{Fragebogen zum Trinkverhalten}

Der Fragebogen zum Trinkverhalten wurde im Rahmen der Diplomarbeit von Andrea Sauer (Sauer 2014) konzipiert und validiert. Er bestand aus einer Sieben-Punkte-Likert-Skala mit „nie“ bis „,sehr oft“. Gefragt wurde nach dem Konsum alkoholischer Getränke, äquivalent zu den Subkategorien der Bilder (Bier-, Wein- und Schnapssorten). Für die abstinenten Patienten wurde die Ausgangsfrage des Bogens in der Vergangenheitsform gestellt. Auch hier wurde die Auswertung mittels SPSS durchgeführt. Bei den Kontrollen wurde nach dem Ausfüllen des Fragebogens am MR-Messungstag auf Tendenzen zum Subalkoholismus geachtet. Bei Verdacht wäre ein Ausschluss aus der Studie erfolgt. 


\subsubsection{Funktionelle Bildgebung}

Die funktionelle Magnetresonanztomographie (fMRT) ist derzeit die Hauptsäule der Hirnbildgebung in der kognitiven Neurowissenschaft (Logothetis 2008). Sie basiert auf dem von Ogawa entdeckten BOLD-Effekt (blood oxygen level dependency) (Ogawa et al. 1990). Logothetis und Kollegen beobachteten eine direkte Korrelation zwischen der neuronalen Aktivität und dem BOLD-Signal im Gehirn (Logothetis 2008).

Die neuronale Aktivität führt $\mathrm{zu}$ einem erhöhten Sauerstoffbedarf und zunächst zu einer erhöhten Desoxyhämoglobin-Konzentration. Als Reaktion kommt es in den aktivierten Arealen zu einem erhöhten zerebralen Blutfluss, was zu einem Überangebot an Sauerstoff in besagten Arealen führt. Physikalisch gesehen besitzt Desoxyhämoglobin vier ungepaarte Elektronen und so einen signifikanten magnetischen Moment (paramagnetisch). Im Gegensatz dazu besitzt Oxyhämoglobin keine ungepaarten Elektronen und ist somit diamagnetisch. Das paramagnetische Desoxyhämoglobin führt zu einer Abnahme der Relaxationsszeit in T2*- Gewichtung bzw. einer Verminderung des MRSignals und umgekehrt. Der Anstieg von Oxyhämoglobin führt so zu einer Zunahme des MR-Signals, was als BOLD-Effekt bezeichnet wird (Ogawa et al. 1990).

Die Versuche wurden am MR-Tomographen der Forschungsgruppe in der Neurologie und Psychiatrie der Universitätsmedizin Göttingen, Abteilung kognitive Neurologie, (Leiter PD Dr. Dechent) durchgeführt. Es kam ein drei Tesla Siemens Magnetom TIM Trio (Erlangen) zum Einsatz. Es wurde eine 8-Kanal-Spule verwendet. Zuerst wurde eine T1-gewichtete anatomische 3D-Messung als Referenz aufgenommen (176 Schichten mit 1 mm Schichtdicke, Time Repetition (TR) $2250 \mathrm{ms,}$ Time Echo (TE) 3,29 ms, Voxelgröße 1 x 1 x 1 mm3, Flip angle $9^{\circ}$, Field of view $256 \mathrm{~mm}$ ). Danach erfolgten drei funktionelle Messungen mit jeweils 465 Einzelbildern. Dazu wurde eine Echo-Planar Imaging (EPI) 
Sequenz verwendet (TR 1900 ms, TE 30 ms, 36 Schichten mit einer Voxelgröße $3 \times 3 \times 3$ mm3, 20\% Gap, Flip angle 70, 36 Schichten. Eine Messung dauerte ca. 50 Minuten. Als letztes wurde eine funktionelle Resting State Messung durchgeführt (TR 2000 ms, TE 30 ms, Flip angle $70^{\circ}, 20 \%$ Gap, 33 Schichten, 156 Einzelbilder). Die Resting State Daten wurden für die Studie nicht ausgewertet.

Der Kopf der Probanden wurde mittels Schaumstoffkeilen immobilisiert, um Bewegungsartefakten vorzubeugen. Die Stimuli wurden den Probanden im Scanner mittels einer Brille präsentiert (VisuaStim Digital, Resonance Technology, Inc., CA, USA). Eine Sehschwächenkorrektur von +3 bis -9 Dioptrien war möglich. Die Probanden bekamen eine Antwortbox („Fiber optic response pad“, Current designs Inc., Philadelphia, USA) in die rechte Hand. Die Antworten wurden aufgezeichnet und in Logfiles gespeichert.

\subsubsection{Statistische Analyse der Verhaltensdaten}

Die Verhaltensdaten wurden mittels SPSS Statistics Version 19 (IBM, NY, USA) ausgewertet. Dafür wurden die von Presentation erstellten Logfiles (Aufzeichnung der Reaktionszeiten und Antworten) mit der SPSS-Software ausgewertet. Die Analyse der Reaktionszeiten auf die unterschiedlichen Stimuli, sowie der Fehlerraten erfolgte mittels einer einfaktoriellen ANOVA. Die Daten zum Trinkverhalten wurden mit nichtparametrischen Tests ausgewertet, Median- und Kruskal-Wallis Test. Für die Verhaltensdaten wird ein Signifikanzniveau von $p<0,05$ verwendet.

\subsubsection{Statistische Analyse der fMRT-Daten}

Die Analyse der fMRT-Daten erfolgte mit SPM12 (Wellcome Department of Cognitive Neurology, London, UK) und MATLAB Version 2014b (Mathworks, Inc., MA, USA). Im Rahmen der Vorverarbeitung (Preprocessing) wurde die Korrektur der Kopfbewegungen („Realigment“) 
und die zeitliche Korrektur der einzelnen EPI-Schichten („Slice Timing") durchgeführt. Im Anschluss erfolgte die räumliche Normalisierung („Spatial Normalization“) in den MNI Standardraum und die Daten wurden mit einer Voxelgröße von 2 x 2 x 2 mm3 gespeichert. Zum Schluss erfolgte die räumliche Glättung („Spatial Smoothing“) mit einem Wert von $6 \times 6 \times 6 \mathrm{~mm} 3$ full width at half maximum (FWHM).

Die statistische Auswertung für die First-Level-Analyse erfolgte mit einem allgemeinen linearen Model (GLM), in das die sieben experimentellen Bedingungen als Regressoren eingingen. Die Bedingungen für GLM ergaben sich aus der Paarung der gezeigten Bilder. Insgesamt waren es sieben verschiedene Kombinationen. Bei allen Kombinationen mit nicht konditioniertem-/ alkoholischem Non-Target, NT, ALKNT durfte frei nach Präferenz gewählt werden. Bei der Kombination von NT/ALKNT mit Target, $\mathrm{T}$, musste immer $\mathrm{T}$ angenommen werden um das Aufgabenziel von 50 Punkten am Ende jenes Blocks zu erreichen. Bei der Kombination von konditionierten Non-Target KNT mit NT durfte KNT angenommen werden und damit 10 zusätzliche Punkte zu erhalten. Letztlich bei der Kombination von KNT mit T musste KNT zugunsten von einem langfristigen Ziel abgelehnt bzw. das T angenommen werden. Die Kombination von Objekten OBJ galt als Kontrollbedingung. Der Blockhinweisreiz (Cue) und das Blockfeedback gingen als weitere Regressoren ein, was zu insgesamt 9 Regressoren führte. Der Vektor des zeitlichen Beginns der Bedingung (onset), wurde mit der kanonischen Hemodynamic Response Function (HRF) gefaltet. Es wurden lineare T-Kontraste definiert, um die differentiellen Effekte, die durch die experimentellen Bedingungen hervorgerufen wurden, zu bewerten (s. Anhang). Die Gruppeneffekte wurden mit Random-Effekts-Analysen beurteilt. Dafür gingen auf Second-Level für jede Bedingung die jeweiligen Kontrast-Daten der First-Level-Analyse in ein GLM ein. Mit einem Two-Sample-T-Test wurden die Effekte auf Gruppenebene verglichen. Die statistischen Effekte wurden auf ein Signifikanzniveau von $\mathrm{p}<0,001$ 
unkorrigiert berichtet. Für Hirnregionen bei denen eine a prioriHypothesen vorliegt, erfolgte eine small volume correction (Worsley et al. 1996). Wir erwarteten einen erhöhten Einfluss der alkoholischen Stimuli bei Alkoholpatienten auf die Funktion des mesolimbischen Dopaminsystems. Die MNI-Koordinaten für small volume correction wurden aus Diekhof und Gruber 2010 verwendet (Diekhof und Gruber 2010), für den NAc R $[12 ; 12 ;-4]$, NAc L $[-12 ; 12 ;-4]$ und die VTA $[8 ;-20 ;-16]$. 


\section{ERGEBNISSE}

\subsection{Fragebögen}

\subsubsection{Visuelle Analogskala für Substanz-Craving (VAS)}

Alle Probanden füllten vor und nach dem Experiment eine visuelle Analogskala für Substanz-Craving aus. Die Skala enthielt zwei Fragen anhand derer die Probanden auf einer Skala von o bis 100 a) ihren aktuellen Suchtdruck (Craving) einschätzen sollten und b) wie gut sie diesen kontrollieren konnten. Der t-Test bei zwei unabhängigen Stichproben zeigte keinen signifikanten Unterschied im Suchtdruck bei Patienten und Kontrollen vor und nach dem Experiment (siehe Tabelle 2).

Tabelle 2: Suchtdruck (Craving) und Kontrolle vor und nach dem Experiment bei Kontrollen und Patienten. t-Test bei unabhängigen Stichproben

\begin{tabular}{|l|l|c|c|c|c|}
\hline \multicolumn{2}{|c|}{} & T & $\begin{array}{c}\text { Sig. } \\
\text { (2-seitig) }\end{array}$ & $\begin{array}{c}\text { MW } \\
\text { Patient }\end{array}$ & $\begin{array}{c}\text { MW } \\
\text { Kontrolle }\end{array}$ \\
\hline $\begin{array}{l}\text { Vor dem } \\
\text { Experiment }\end{array}$ & Suchtdruck (Craving) & 1,669 & 0,106 & 11,76 & 2,00 \\
\cline { 2 - 6 } & Kontrolle & $-1,145$ & 0,261 & 93,82 & 99,00 \\
\hline $\begin{array}{l}\text { Nach dem } \\
\text { Experiment }\end{array}$ & Suchtdruck (Craving) & 1,421 & 0,166 & 11,18 & 2,67 \\
\cline { 2 - 6 } & Kontrolle & $-1,266$ & 0,215 & 93,24 & 99,67 \\
\hline
\end{tabular}

Auch führte die Teilnahme am Experiment nicht zu erhöhtem Suchtdruck, bzw. zu einem Unterschied in seiner Kontrolle, siehe Tabelle 3.

Tabelle 3: Suchtdruck (Craving) und Kontrolle vor und nach dem Experiment, $t$-Test bei gepaarten Stichproben

\begin{tabular}{|l|l|c|c|c|}
\hline \multicolumn{2}{|c|}{} & T & $\begin{array}{c}\text { Sig. } \\
\text { (2-seitig) }\end{array}$ & MW \\
\hline $\begin{array}{l}\text { Vor und nach dem } \\
\text { Experiment }\end{array}$ & Suchtdruck (Craving) & 0,000 & 1,000 & 0,000 \\
\cline { 2 - 5 } & Kontrolle & 0,000 & 1,000 & 0,000 \\
\hline
\end{tabular}




\subsubsection{Fragebogen zum Trinkverhalten}

Die Sieben-Punkt-Likert-Skala mit „nie“ bis „sehr oft" fragte nach dem Konsum alkoholischer Getränke, äquivalent zu den Subkategorien der Bilder (Bier-, Wein- und Schnapssorten). Für die abstinenten Patienten wurde die Ausgangsfrage des Bogens in Vergangenheitsform gestellt. Die Auswertung des Fragebogens erfolgte mit nicht parametrischen Tests für unabhängige Stichproben. Verglichen wurden die Gruppen und die Geschlechter untereinander mittels Median- und Kruskal-Wallis Test. Einerseits sollte das Trinkverhalten unter Alkoholpatienten und gesunden Kontrollen verglichen werden, anderseits wollten wir wissen, welches Geschlecht mehr Alkohol konsumiert. Hierbei konnte kein signifikanter Unterschied festgestellt werden. Bei den Kontrollen wurde nach dem Ausfüllen des Fragebogens am MR-Messungstag auf Tendenzen zum Subalkoholismus geachtet. Bei Verdacht wäre der Proband aus der Studie ausgeschlossen worden.

Tabelle 4: Unterschiede im Trinkverhalten zwischen Patienten und Kontrollen bzw. Männern und Frauen. Nicht parametrischer Test für unabhängige Stichprobe auf Gruppenebene und für das Geschlecht

\begin{tabular}{|l|l|c|}
\hline \multirow{2}{*}{$\begin{array}{l}\text { Trinkverhalten } \\
\text { Patienten vs. Kontrollen }\end{array}$} & \multicolumn{1}{|c|}{ Test } & Sig. \\
\hline \multirow{2}{*}{$\begin{array}{l}\text { Trinkverhalten } \\
\text { Männlich vs. Weiblich }\end{array}$} & Kruskal-Wallis & 0,306 \\
\cline { 2 - 3 } & Median & 0,181 \\
\cline { 2 - 3 } & Kruskal-Wallis & 0,673 \\
\hline
\end{tabular}

Statistische Effekte bei einem Signifikanzniveau von $p<0,05$. 


\subsection{Verhaltensdaten}

Die statistischen Analysen zeigten eine signifikant erhöhte Antwortzeit der Patienten im Vergleich zu Kontrollen in allen Bedingungen, in denen alkoholische Stimuli in Kombination mit anderen Stimuli angeboten wurden. In der Situation, wenn ALKNT zugunsten eines langfristiges Zieles abgelehnt wurde (Target alk) und bei freier Wahl zwischen ALKNT und NT (Alk rej), brauchten Patienten signifikant mehr Zeit, um alkoholische Stimuli abzulehnen. Im Desire-Kontext, wenn konditionierte Stimuli angenommen werden durften (Reward), brauchten beide Gruppen bei der Entscheidungsfindung am längsten. Insgesamt brauchten die Patienten in allen berichteten Bedingungen im Vergleich zu Kontrollen signifikant mehr Antwortzeit.

Tabelle 5: Signifikante Differenzen der Antwortzeiten zwischen Patienten und Kontrollen während der Messung im MRT. Einfaktorielle ANOVA

\begin{tabular}{|l|c|c|c|c|}
\hline \multicolumn{1}{|c|}{ Bedingung } & Kontrollen & Patienten & F & Signifikanz \\
\hline Alk_rej & 837,6 & 966,3 & 4,38 & P \\
\hline Reason & 733,2 & 841,5 & 5,38 & 0,045 \\
\hline Reward & 939,8 & 1040,8 & 4,4 & 0,027 \\
\hline Target & 709,8 & 798,5 & 4,99 & 0,033 \\
\hline Target_alk & 641,9 & 750,8 & 9,44 & 0,004 \\
\hline $\begin{array}{l}\text { MW aller } \\
\text { Antworten }\end{array}$ & 840,5 & 929,6 & 3,79 & 0,061 \\
\hline
\end{tabular}


Im Desire-Kontext durften zuvor operant konditionierte Belohnungsreize angenommen werde, um 10 zusätzliche Punkte zu erzielen. In dieser Situation haben die Kontrollen im Vergleich zu Patienten signifikant mehr belohnende Stimuli angenommen. Die Patienten haben dagegen signifikant mehr belohnende Stimuli nicht angenommen.

Tabelle 6: Vergleich der angenommenen und nicht angenommenen konditionierten Stimuli unter den beiden Gruppen

\begin{tabular}{|l|l|c|c|}
\hline \multirow{2}{*}{ Reward_Anzahl } & Kontrolle & MW & Signifikanz \\
\cline { 2 - 4 } & Patient & 35,73 & 0,019 \\
\hline \multirow{2}{*}{ Reward_miss_Anzahl } & Kontrolle & 2,75 & 0,019 \\
\cline { 2 - 4 } & Patient & 9,13 & \\
\hline
\end{tabular}

Bezüglich der Anzahl der angenommenen Stimuli gab es bis auf die Anzahl der alkoholischen Bilder keine signifikanten Unterschiede. Aber es haben auch nur jeweils 7 Probanden aus jeder Gruppe diese Stimuli überhaupt angenommen. Alle anderen Stimuli wurden von allen Probanden angenommen.

Tabelle 7: Anzahl der angenommenen alkoholischen Stimuli im Vergleich zu den anderen Stimuli, in beiden Gruppen

\begin{tabular}{|l|c|c|c|c|c|}
\hline & Kontrollen & Patienten & F & Signifikanz & \\
\hline & \multicolumn{3}{|c|}{ Mittelwert angenommene Stimuli } & \\
\hline Alkohol & 7,1 & 21,1 & 5,42 & 0,038 & $\begin{array}{c}\mathrm{N}=7 \text { für jede } \\
\text { Gruppe, alle } \\
\text { anderen haben } \\
\text { keine Alk.-Bilder } \\
\text { angenommen }\end{array}$ \\
\hline
\end{tabular}




\subsection{Funktionelle Bildgebung}

Da es sich in dieser Studie um den ersten Einsatz des DRD-F10- Paradigmas handelte, wurde für die Kontrollgruppe überprüft, ob die konfinierten Stimuli zu einer Bottom-Up-Aktivierung in VTA und NAc führen und ob deren Suppression im Reason-Kontext auftrat. Sowohl die Bottom-Up-Aktivierung als auch die Top-Down-Suppression konnten in VTA und NAc beobachtet werden, wobei sie nicht immer small volume-korrigiert signifikant waren, was auch auf die etwas kleine Kontrollgruppe ( $\mathrm{N=15}$ ) zurückzuführen ist (s. Anhang Tabelle 8 ).

\subsubsection{Unterschiede bei konditionierten nicht alkoholischen Stimuli}

Im Desire-Kontext sollten die konditionierten Stimuli angenommen werden. Abstinente Alkoholpatienten zeigten hierbei eine reduzierte Aktivierung in Bereich des erweiterten Belohnungssystems. Bei gesunden Kontrollen wurde im Vergleich mit Patienten eine erhöhte Aktivierung, vor allem im ventralen Striatum $(t=3,66, p<0,001[4 ; 18 ;-$ $2])$, anterioren frontoorbitalen Kortex $(t=4,90, p<0,001[26 ; 58 ;-14])$, Hypothalamus $(\mathrm{t}=3,13, \mathrm{p}<0,001[-10 ; 14 ;-6])$ und linken Hippocampus $(\mathrm{t}=4,25, \mathrm{p}<0,001[-28 ;-16 ;-10])$ beobachtet. 
A

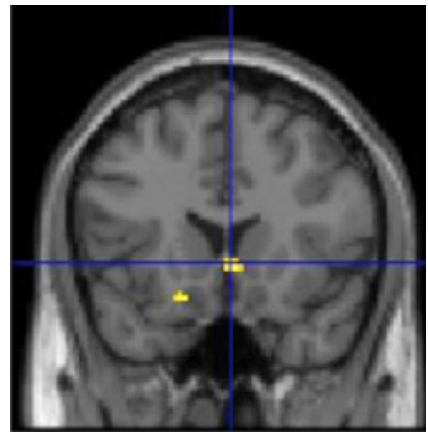

c

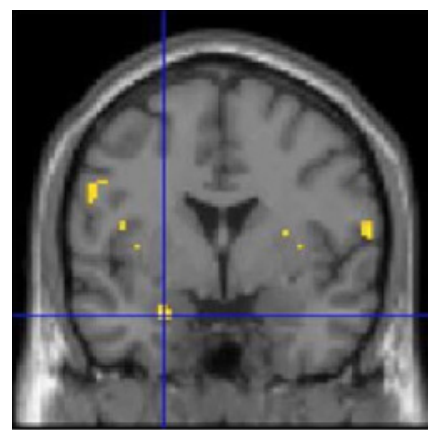

B

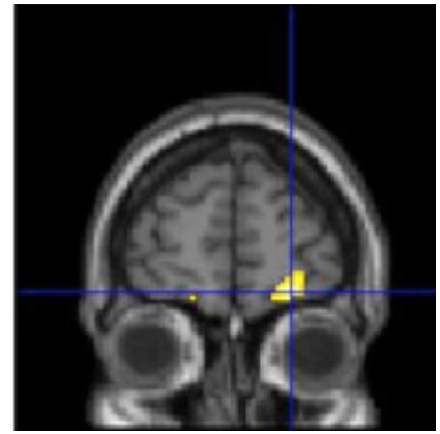

D

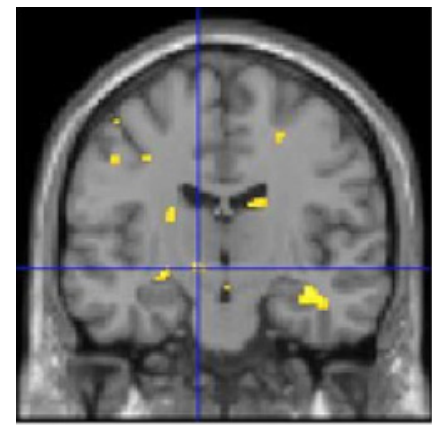

Abbildung 3: Reduzierte Aktivierung auf konditionierte Belohnungsreize bei Alkoholpatienten: Kontrollen zeigten im Vergleich zu Alkoholpatienten eine erhöhte Aktivierung im A) VSt $(t=3,66, p<0,001[4 ; 18 ;-2]), B)$ anterioren frontoorbitale Kortex $(t=4,90, p<$ $0,001[26 ; 58 ;-14]), C)$ linkem HPC $(t=4,25, p<0,001[-28 ;-16 ;-10])$ und D) Hypothalamus $(t=3,13, p<0,001[-10 ; 14 ;-6])$.

Andererseits zeigten die Patienten im Vergleich zu den Kontrollen eine verstärkte Antwort auf konditionierte Belohnungsreize im prägenualen ACC $(t=3,395, P<0,001[-6 ; 52 ; 16])$ und tiefen frontalen Operculum/ anteriore Insel $(\mathrm{t}=3,685, \mathrm{P}<0,001[-38 ; 22 ;-12])$ (s. Abb. 4). Darüber hinaus wurde bei Patienten eine verminderte Top-down-Suppression von Belohnungsreizen im NAc beobachtet. In diesem Kontext zeigten die Kontrollen eine erhöhte Aktivierung im ventralen Striatum $(t=4,12$, $\mathrm{p}<0,005[2 ; 18 ;-4])$ (s. Abb. 5). 
A
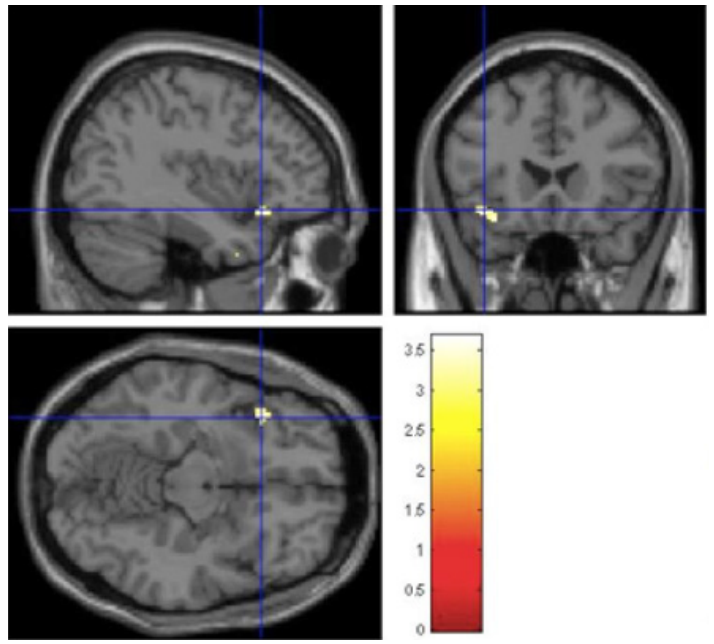

B
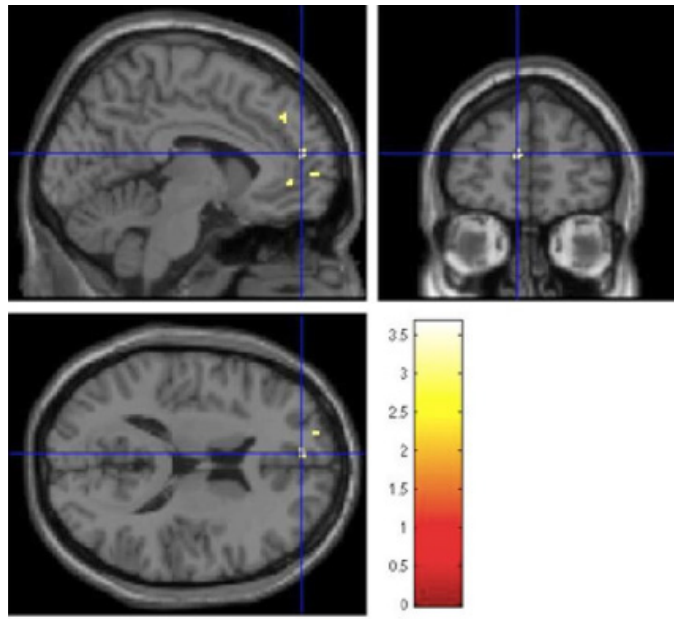

Abbildung 4: Verstärkte Aktivierung auf konditionierte Belohnungsreize bei Alkoholpatienten: Patienten zeigten im Vergleich zu Kontrollen eine verstärkte Aktivierung in A) tiefes frontales Operculum/ anteriore Insel $(t=3,685, P<0,001[-38 ; 22 ;-12])$ und $B)$ $\operatorname{pgACC}(t=3,395, P<0,001[-6 ; 52 ; 16])$. 


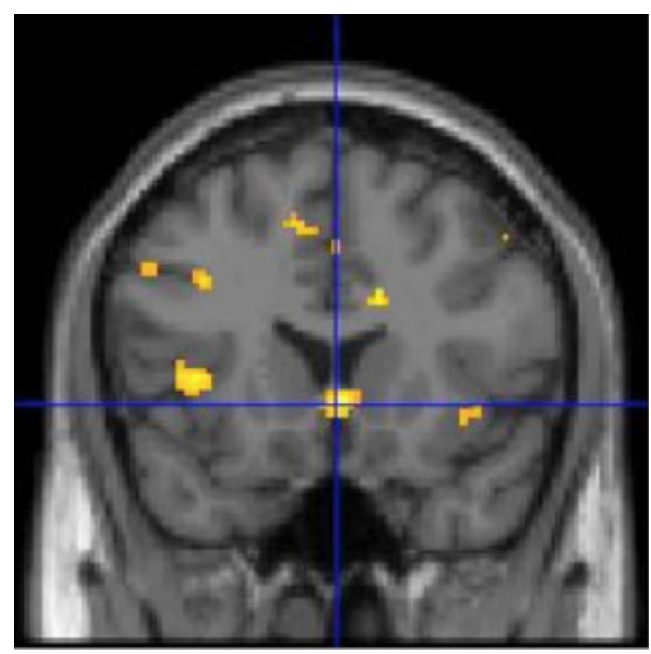

Abbildung 5: Verminderte Top-down-Suppression von Belohnungssignalen im N. accumbens bei Alkoholpatienten: Kontrollen zeigten eine erhöhte Aktivierung in VSt $(t=4,12, p<$ $0,005[2 ; 18 ;-4])$.

\subsubsection{Unterschiede bei alkoholischen Stimuli}

Bei der Auswahl von alkoholischen Stimuli zeigten Alkoholpatienten eine erhöhte Aktivierung in der rechten VTA $(t=2,13, p<0,05[10 ;-18 ;-$ 12]) und im posterioren $\mathrm{OFC}(\mathrm{t}=3,58, \mathrm{p}<0,001[-30 ; 24 ;-16])$. Gesunde Kontrollen hingegen zeigten eine erhöhte Aktivierung im rechten VSt $(\mathrm{t}=4,29, \mathrm{p}<0,001[8 ; 18 ;-8]), \operatorname{pgACC}(\mathrm{t}=3,41, \mathrm{p}<0,001[8 ; 46 ; 2])$ und im subgenualen ACC (sgACC) $(t=3,29, p<0,001[0 ; 34 ;-14])$. 
A
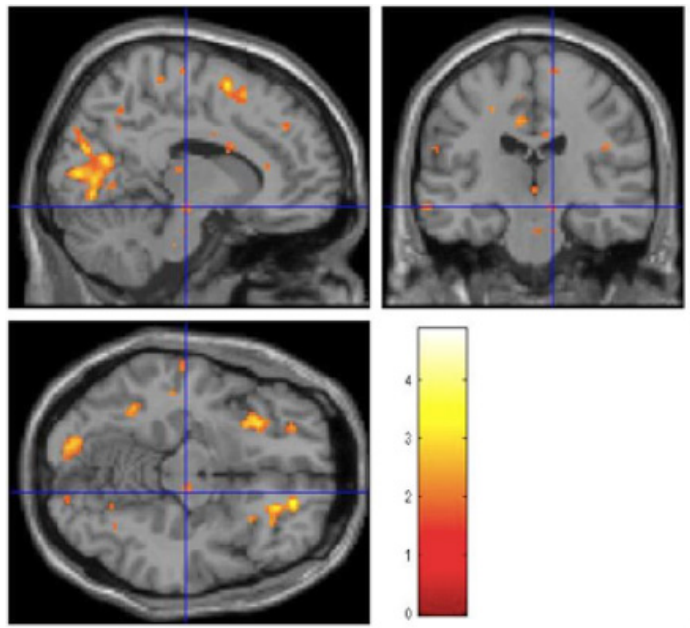

B
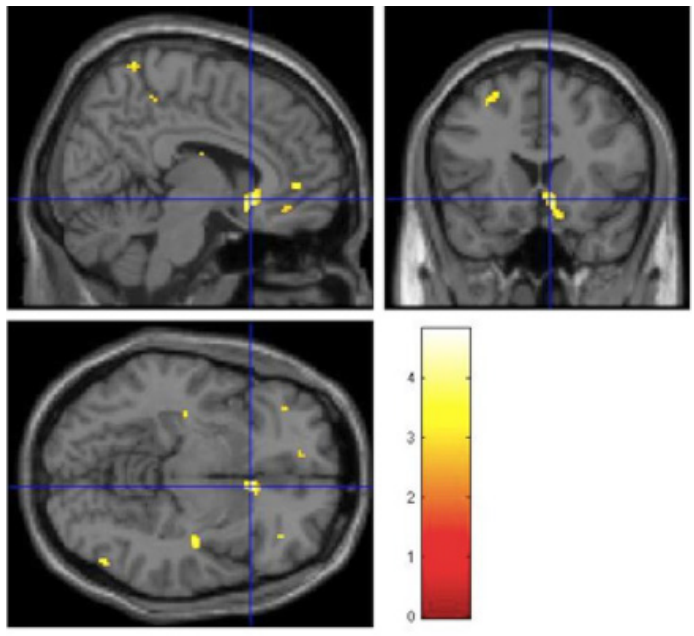

Abbildung 6: Veränderte Aktivierung auf alkoholischen Stimuli bei Alkoholpatienten: Patienten zeigten bei Auswahl von alkoholischen Stimuli eine erhöhte Aktivierung in A) rechten VTA $(t=2,13, p<0,05[10 ;-18 ;-12])$ und posterioren OFC $(t=3,58, p<0,001[-30 ; 24 ;-$ 16]). Kontrollen hingegen zeigten bei Auswahl von alkoholischen Stimuli eine erhöhte Aktivierung im $B)$ rechten VSt $(t=4,29, p<0,001[8 ; 18 ;-8])$, pgACC $(t=3,41, p<0,001[8 ; 46 ; 2])$ und den $\operatorname{sgACC}(t=3,29, p<0,001[0 ; 34 ;-14])$

\subsubsection{Top-Down-Suppression in Desire-Reason-Dilemma-Situation und bei Ablehnung von alkoholischen Stimuli}

Im Reason-Kontext, wenn unmittelbare Belohnung zugunsten eines langfristigen Ziels abgelehnt werden sollte, zeigten abstinente Alkoholpatienten einen Suppressionseffekt des erweiterten Belohnungssystems. 
Im Vergleich dazu zeigten sie bei Ablehnung der alkoholischen Stimuli einen signifikant stärkeren Supprimierungseffekt in der VSt $(t=4,127$, $\mathrm{p}<0,001[2 ; 18 ;-4])$, dem linken posterioren $\mathrm{OFC}(\mathrm{t}=4,471, \mathrm{p}<0,001$ $[-26 ; 26 ;-18])$ und dem linken VTA $(t=3,701, p<0,001[-4 ;-26 ;-12])$.

A
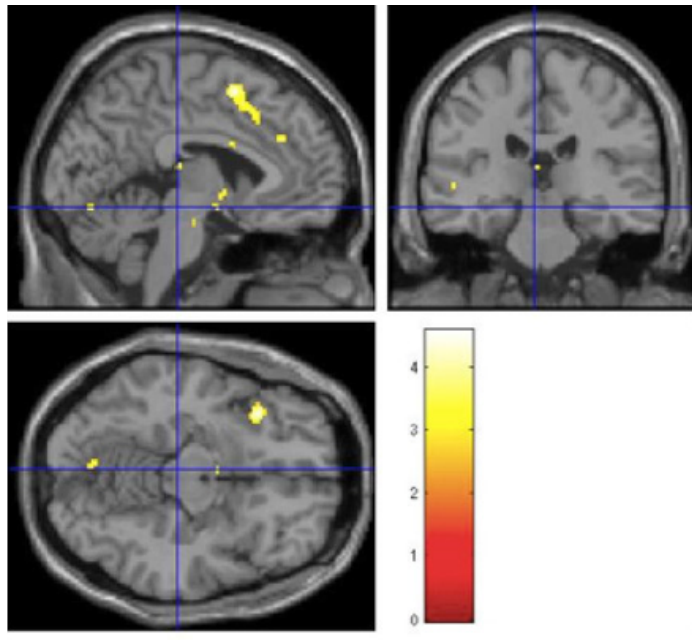

B
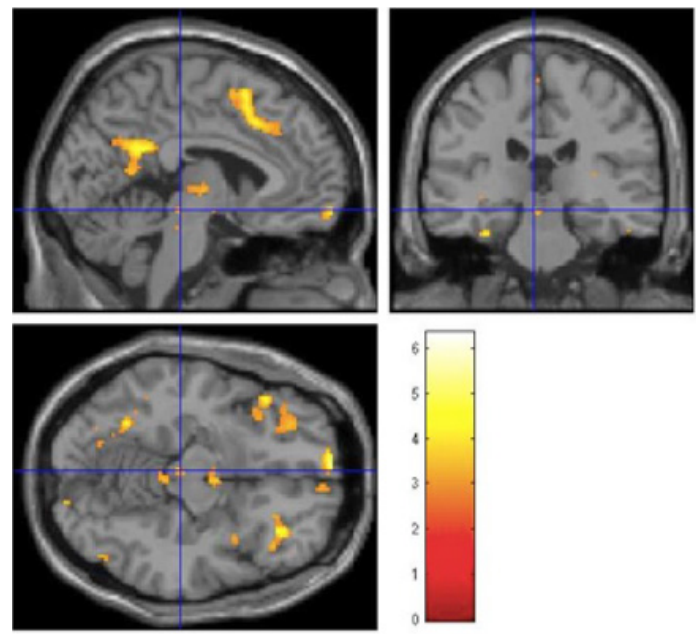

Abbildung 7: Vergleich der Top-Down-Suppression auf konditionierte Belohnungsstimuli zu alkoholischen Stimuli bei Alkoholpatienten: Konditionierte Stimuli führtenin DRD-Situation zu einem A) Suppressionseffekt in erweiterten Belohnungssystem. Ablehnung von alkoholischen Stimuli hingegen führten zu einem signifikant stärkeren Suppressionseffekt im B) VSt $(t=4,127, p<0,001[2 ; 18 ;-4])$, linken posterioren OFC $(t=4,471, p<0,001[-$ $26 ; 26 ;-18])$ und den linken VTA $(t=3,701, p<0,001[-4 ;-26 ;-12])$. 


\section{DISKUSSION}

Das Ziel dieser Studie war es, mittels funktioneller Kernspintomographie potentielle Dysfunktionen neurofunktioneller Systeme der Impulskontrolle bei Patienten mit langjähriger Alkoholsucht zu untersuchen. Dazu wurde das Paradigma DRD-F10 auf Basis des DRD-Paradigmas weiterentwickelt, um die präfrontale Modulation des Belohnungssystems bewerten zu können, während abstinente Alkoholpatienten und Kontrollprobanden sich zwischen unmittelbarer und langfristiger Belohnung entscheiden mussten (Diekhof und Gruber 2010; Diekhof et al. 2012a).

In der Situation, wenn unmittelbare Belohnungsreize bzw. konditionierte Stimuli im Desire-Kontext (s. 2.2.1) angenommen werden durften, zeigten abstinente Alkoholpatienten eine signifikant reduzierte Aktivierung des VSt und des frontoorbitalen Kortex. Im NAc zeigten sie eine verminderte Top-Down-Suppression von Belohnungsreizen. $\mathrm{Zu}$ sätzlich wurde bei ihnen eine signifikante Reduktion der Aktivität im lateralen Hypothalamus (LHA) und im HPC beobachtet. Diese Areale gehören nachgewiesenermaßen zu den Schlüsselstrukturen des Belohnungssystems und haben eine zentrale Rolle bei der Belohnungsverarbeitung (Haber und Knutson 2010). Letztlich wurde bei den Patienten eine signifikant erhöhte Aktivität im prägenualen ACC und im tiefen frontalen Operculum/anteriore Insel beobachtet, die ebenfalls an der Belohnungsverarbeitung beteiligt sind (Gruber et al. 2009; Haber und Knutson 2010; Cao et al. 2018).

Das Ergebnis, dass entgiftete Alkoholiker geringere Aktivierungen im Belohnungssystem in Reaktion auf belohnte Stimuli aufweisen, steht im Einklang mit den Befunden früherer Studien. So wurde bei entgifteten Alkoholikern (Wrase et al. 2007; Beck et al. 2009), bei Substanzabhängigen und bei Spielsuchtpatienten (Luijten et al. 2017), im Vergleich zu gesunden Kontrollen eine reduzierte Aktivierung des VSt 
während der Antizipation von unmittelbarer Belohnung festgestellt. Eine mögliche Ursache für diesen Befund könnte die Veränderung des dopaminergen Belohnungssystems bei chronischem Alkoholmissbrauch sein (Koob 2013). Durch die Kombination aus D2-Rezeptordichte (Volkow et al. 2002; Heinz et al. 2004) und reduzierter Dopaminausschüttung (Volkow et al. 2007) kann es zu einer verminderten Empfindlichkeit der Belohnungsareale kommen (Volkow und Fowler 2000; Martin-Sölch et al. 2001). Eine zweite mögliche Ursache dafür könnte die Ablenkung bzw. Aufmerksamkeitsverlagerung der Patienten durch die attraktiveren bzw. salienten alkoholischen Stimuli sein (Wrase et al. 2007). Die verminderte Top-Down-Suppression der Patienten im NAc, einem wichtigen Bestandteil des Belohnungssystems (Diekhof und Gruber 2010), ist sehr wahrscheinlich durch die verminderte Aktivierung des Belohnungssystems bedingt, da aufgrund der geringeren Aktivität in diesen Arealen auch deren Suppression nicht so stark ausgeprägt ist.

Während der Präsentation vom alkoholischen Non-Target zeigten abstinente Alkoholpatienten im Vergleich zu gesunden Kontrollen erwartungsgemäß signifikant erhöhte Aktivierungen der VTA und dem posterioren OFC. Die VTA als wichtiger Bestandteil des dopaminergen Belohnungssystems erfüllt eine modulatorische Funktion durch Verschaltungen mit anderen Arealen des limbischen Systems. Daneben bewirkt sie eine positive Verstärkung des Verhaltens (Oades und Halliday 1987; Knutson et al. 2001; O'Doherty 2004) und eine zentrale Rolle bei Bewertung des Anreizwerts (Salienz) suchtmittelassoziierter Stimuli (Berridge 2007). Der OFC unterstützt zielgerichtetes Verhalten und reflektiert die Belohnungsvorhersage (Schoenbaum et al. 1998; Tremblay und Schultz 1999; O'Doherty et al. 2002). Im Rahmen von weiteren Studien wurden der posteriore OFC und der vmPFC in Verbindung mit Selbstkontrolle, Entscheidungsfindung und Belohnungswert gebracht (Diekhof et al. 2012b; Hebscher et al. 2016), welche 
bekannterweise bei Suchtpatienten gestört sind.

Der Befund einer verstärkten Reaktion auf alkoholische Stimuli in der Alkoholgruppe ist konsistent mit Befunden zahlreicher Studien, die die Salienz suchtpotenter Rauschmittel (Zijlstra et al. 2009), darunter auch Alkohol, sowohl bei chronischem Missbrauch bzw. Abhängigkeit als auch die Auswirkungen auf das dopaminerge Belohnungssystem bei Tieren (Gessa et al. 1985; Brodie et al. 1990) und abstinenten Alkoholikern (Wrase et al. 2002; Wrase et al. 2007; Heinz et al. 2004; Kareken et al. 2004) untersucht haben.

Die Incentive Sensitization Theory of Addiction von Berridge und Robinson beschreibt die neuro- und verhaltensbiologischen Zusammenhänge. Sie geht von einer Sensitivierung mesolimbischer Areale, unter anderem auch der VTA und dem OFC, durch suchtpotente Substanzen aus. Diese neuroadaptiven Prozesse führen dazu, dass die suchtmittelassoziierten Stimuli im Vergleich zu natürlichen Belohnungsreizen einen höheren Anreizwert (Salienz) besitzen und im Verlauf der Suchtentwicklung sich dieser Anreizwert steigert (sensitization of incentive salience). Jedoch bleibt der hedonische Wert konstant oder nimmt sogar ab (Robinson und Berridge 1993; Berridge und Robinson 2003).

Bei Auswertung der Verhaltensdaten verglichen wir Patienten versus Kontrollen hinsichtlich Reaktionszeit auf unmittelbare Belohnung und alkoholische Stimuli.

Diese Daten stützen die zwei oben aufgeführten Befunde. Bei der Ablehnung von alkoholischen Stimuli brauchten die abstinenten Alkoholpatienten im Vergleich zu den gesunden Kontrollen signifikant mehr Zeit.

Außerdem haben die Patienten bei der Auswahl von konditionierten Stimuli signifikant weniger Stimuli angenommen (s. 3.2).

In der Desire-Reason-Dilemma-Situation beobachteten wir bei abstinenten Alkoholpatienten, wie erwartet, einen Top-Down-Suppressionseffekt im dopaminergenen Belohnungssystem. Hierbei wurden 
im Reason-Kontext die zuvor belohnten/konditionierten Stimuli KNT zugunsten eines langfristigen Ziels abgelehnt. Eine Top-Down-Suppression der Belohnungsareale bei Ablehnung konditionierter Stimuli war bereits in Vorstudien der Arbeitsgruppe bei gesunden Probanden beobachtet worden (Diekhof und Gruber 2010). Präfrontale Areale wurden als modulierende Schlüsselstrukturen bei der Handlungskontrolle, insbesondere bei Beurteilung langfristiger Nachteile einer Handlung, identifiziert (Daw et al. 2005; Daw et al. 2006; Kim et al. 2017). Letztlich wurde in der Alkoholgruppe ein Vergleich der Top-Down-Suppression in der Dilemma-Situation mit der Top-Down-Suppression bei Ablehnung von alkoholischen Stimuli ALKNT durchgeführt. Zum ersten Mal haben wir im Rahmen unserer Studie bei Alkoholpatienten eine erhöhte Top-Down-Suppression des dopaminergen Belohnungssystems, in VTA, VSt und posteriore OFC, während der Ablehnung von alkoholischen Stimuli zugunsten eins langfristigen Ziels beobachtet. Diese experimentelle Situation hatte große Ähnlichkeit mit realen Situationen im Leben der Suchtpatienten, in denen der kurzfristigen Versuchung das Suchtmittel zu konsumieren widerstanden werden muss, um langfristig die Lebensqualität aufrechtzuerhalten. Alkoholpatienten waren in dieser Situation in der Lage, eine erhöhte Aktivität des erweiterten Belohnungssystems zu supprimieren. Impulsives Handeln wurde unterdrückt, um das langfristige Ziel im Rahmen des Experiments zu erreichen. Diesen Effekt bei Patienten interpretieren wir als einen erfolgreichen Therapieeffekt nach der stationären Entzugsbehandlung.

Da die Gefahr bestand, dass die im MR verwendeten alkoholischen Stimuli bei abstinenten Alkoholpatienten unter Umständen den Suchtdruck erhöhen, wurde, wie im Kapitel Methoden beschrieben, nach der MR-Messung eine außerordentliche therapeutische Sitzung durchgeführt. Zusätzlich wurde bei allen Probanden eine durch Wedekind und Kollegen weiterentwickelte visuelle Analog-Skala (Wedekind et 
al. 2010) jeweils vor und nach der MR-Messung ausgefüllt. Die Ergebnisse zeigten keinen signifikanten Unterschied zwischen den beiden Gruppen bzw. vor- und nach der MR-Messung (s. 3.1.1). In der Alkoholgruppe könnte dieser Befund auf eine erfolgreiche Therapie und in der Kontrollgruppe auf eine adäquate Selbstkontrolle zurückzuführen sein.

Darüber hinaus wurden die Trinkgewohnheiten der Probanden mittels einer Sieben-Punkt-Likert-Skala „nie“ bis „,sehr oft“ ermittelt. Diese war im Rahmen der Diplomarbeit von Andrea Sauer konzipiert und validiert worden (Sauer 2014). Gefragt wurde nach dem Konsum alkoholischer Getränke, äquivalent zur den Subkategorien der Bilder (Bier-, Wein- und Schnapssorten). Für die abstinenten Patienten wurde die Ausgangsfrage des Bogens in Vergangenheitsform gestellt. Auch hier konnte kein signifikanter Unterschied festgestellt werden (s. 3.1.1).

Die allgemeine Aussagefähigkeit der Ergebnisse dieser Studie wird durch den Umstand eingeschränkt, dass die beiden Probandenkohorten für die Variablen Lebensalter, Geschlecht, Bildungsniveau und Nikotinkonsum nicht ausgeglichen werden konnten. Die Probanden aus der Alkoholgruppe befanden sich zum Zeitpunkt der Untersuchung entweder in stationärer Entzugsbehandlung in der Suchtklinik oder in tagesklinischer Behandlung der UMG (mindestens 14 Tage nach der Entzugsbehandlung). In der Kontrollgruppe war es aufgrund der zeitlich begrenzten Studiendauer nur möglich, als Kontrollprobanden überwiegend Studenten zu rekrutieren. Die meisten der rekrutierten Probanden aus der Alkoholgruppe waren männlich, in der Kontrollgruppe waren die beiden Geschlechter ausgewogen verteilt.

Auch das Bildungsniveau war in der Alkoholgruppe durchschnittlich niedrig und das in der Kontrollgruppe durchschnittlich hoch. Die meisten Raucher stammten aus der Alkoholgruppe. Diese Unterschiede können eine Auswirkung auf die Endergebnisse haben.

Diese Studie ist jedoch die erste Untersuchung mit dem neuen 
DRD-F10-Paradigma und musste aufgrund der zeitlichen Beschränkungen unter diesen Randbedingungen durchgeführt werden. Im Rahmen von weiteren klinischen Studien müssen dann Kohorten mit ausgeglichenen demographischen Parametern rekrutiert und die Ergebnisse dieser Studie validiert werden.

In den letzten Jahren hat die Erforschung der Pathogenese von Suchterkrankungen zunehmend an Bedeutung gewonnen. Der Fokus lag hierbei insbesondere auf den neurofunktionellen Systemen, die den kognitiven und affektiven Funktionen zugrunde liegen und bei Suchtpatienten nachgewiesenermaßen gestört sind. Neben Defiziten in der motivationalen Verarbeitung und der Fähigkeit, Verhaltensimpulse zu unterdrücken, sind bei Suchtpatienten relativ unabhängig von Art der Droge Veränderungen in der Funktion und Struktur des PFC, insbesondere des orbitofrontalen und frontomedialen Kortex, sowie dopaminerg innervierter mesolimbischer Areale, z. B. NAc, von Bedeutung (Havemann und Kuschinsky 1982; Kalivas und Volkow 2005; Diekhof et al. 2008).

Zum ersten Mal wurde mittels des Entscheidungsfindungsparadigmas DRD-F10 der Unterschied zwischen Belohnung konditionierter und alkoholischer Stimuli bei abstinenten Alkoholpatienten und gesunden Kontrollen untersucht. Dabei kamen validierte Bilder alkoholischer und nicht-alkoholischer Stimuli zum Einsatz. Die beobachteten Effekte alkoholischer Stimuli stehen im Einklang mit Ergebnissen anderer Arbeitsgruppen, die bei entgifteten Alkoholpatienten die genannten Unterschiede untersucht haben. Neu ist jedoch in unserer Studie die Beobachtung eines stärkeren Top-Down-Suppressionseffekts des mesolimbischen Dopaminsystems in Dilemma-Situation, wenn alkoholische Stimuli zugunsten eines langfristigen Ziels abgelehnt wurden.

Eingeschränkt werden die Ergebnisse durch einen möglichen Alterseffekt. Jedoch deutet die Tatsache, dass die Patienten bei Ablehnung von 
alkoholischen Stimuli mehr Zeit brauchten und dazu die konditionierten Belohnungsstimuli weniger interessant fanden, darauf hin, dass die Unterschiede bei Reaktion auf alkoholische Stimuli in der Patientengruppe auf die Alkoholerkrankung zurückzuführen sind. 


\section{$5 \quad$ AUSBLICK}

Die Suchterkrankungen, darunter auch Alkoholismus weisen Aspekte sowohl von Impulskontrollstörungen als auch von Zwangsstörungen auf. Langjährige Patienten erleben ihren Konsum als zwanghaftes Verlangen (wanting) und unangenehm (Berridge et al. 2009), konsumieren aber trotz des Wissens über die schädlichen Folgen des Alkohols weiter. Auch nach jahrelanger Abstinenz ist die Gefahr eines Rückfalls gegeben und nicht zu unterschätzen. Bei langjährigen Alkoholpatienten wurden zusätzlich alkoholbedingte Hirnatrophie in Bereichen, die mit Verhaltenskontrolle (Beck et al. 2009) und mit prospektivem Rückfallrisiko (Wrase et al. 2008) in Verbindung stehen, beobachtet. Bislang ist jedoch noch wenig über die tatsächlichen Beziehungen zwischen den an Entstehung und Aufrechthaltung der Sucht beteiligten präfronto-mesolimbischen Netzwerken bekannt. Um Diagnose, Therapie und Erhalt der Abstinenz zu verbessern braucht es ein besseres Verständnis der neurofunktionellen Veränderungen in den betroffenen Netzwerken. Mit dem DRD-F10-Paradigma besteht die Möglichkeit, die Modulation von Belohnungssignalen in striatalen Regionen durch Areale des PFC zu untersuchen. Im Rahmen von weiteren klinischen Studien sollten funktionelle Konnektivitätsanalysen durchgeführt werden, um zu bestimmen, ob eine gestörte präfrontale Interaktion mit ventralen striatalen Reaktionen auf suchtassoziierten Stimuli ein Marker für die Suchterkrankung sein kann und ob dieser hirnbildgebende Marker das Risiko für Rückfälle vorhersagen könnte. Außerdem sollte das Experiment mit einer nach demografischen Parametern ausgeglichenen Kohorte wiederholt werden, um die Befunde dieser Studie zu validieren. 


\section{ZUSAMMENFASSUNG}

Die Suchterkrankungen gehören zu einem der größten Problemgebiete des heutigen Gesundheitswesens weltweit. Sie weisen Aspekte sowohl von Impulskontrollstörungen als auch von Zwangsstörungen auf. Areale des mesolimbischen Dopaminsystem spielen bei der Belohnungsverarbeitung eine zentrale Rolle und ermöglichen schnelle (impulsive) Entscheidungen für präferierte (hedone) oder konditionierte Stimuli (Havemann und Kuschinsky 1985). Präfrontale Areale üben wiederum eine Unterdrückung dieses impulsiven Verhaltens zugunsten eines langfristigen Ziels aus.

Alkohol führt bei chronischem Missbrauch zu irreversiblen Veränderungen des mesolimbischen Dopaminsystems. Die Sensitivierung der dopaminergen Neurone führt zu einem höheren Anreizwert (incentive salience) der alkoholassoziierten Stimuli, welcher im Verlauf der Krankheit zunimmt. Jedoch bleibt der hedonische Wert konstant oder nimmt sogar ab (Robinson und Berridge 1993; Berridge und Robinson 2003).

Das Ziel dieser Studie war es, mittels funktioneller Kernspintomographie potentielle Dysfunktionen neurofunktioneller Systeme der Impulskontrolle bei Patienten mit langjähriger Alkoholsucht zu untersuchen. Dazu wurde das Paradigma DRD-F10 auf der Basis des DRD-Paradigmas (Diekhof und Gruber 2010; Diekhof et al. 2012a) weiterentwickelt, um die präfrontale Modulation des Belohnungssystems bewerten zu können, während abstinente Alkoholpatienten und Kontrollprobanden sich zwischen unmittelbarer und langfristiger Belohnung entscheiden mussten. Zu diesem Zweck wurden entgiftete Alkoholsuchtpatienten und gesunde Kontrollen einer fMRT-Untersuchung unterzogen, während sie das speziell für alkoholische Stimuli modifizierte DRD-Paradigma bearbeiteten. Als Stimulusmaterial wurden Bilder von alkoholischen und nicht-alkoholischen Getränken bzw. 
Objekten verwendet, die im Rahmen der Diplomarbeit von Andrea Sauer in der Arbeitsgruppe entwickelt und validiert wurden (Sauer 2014). Als Hauptbefund aktivierten die alkoholischen Stimuli in der Patientengruppe die Areale des dopaminergen Belohnungssystems. Im Desire-Kontext, wenn konditionierte Belohnungsstimuli angenommen werden durften, zeigten die Patienten jedoch eine reduzierte Aktivierung der besagten Areale. Zusammen deuten diese Befunde auf eine höhere Salienz von alkoholassoziierten Stimuli hin. Auch bei der Ablehnung von alkoholischen Stimuli waren die Patienten langsamer. Als Nebenbefund beobachteten wir in der Patientengruppe einen stärkeren Top-Down-Suppressionseffekt, wenn alkoholische Stimuli zugunsten eines langfristigen Ziels abgelehnt wurden. Diesen Befund interpretieren wir als einen erfolgreichen Therapieeffekt nach stationärer Behandlung. 


\section{ANHANG}

\subsection{Tabellen}

Tabelle 1: Demographische Daten, Ein- und Ausschluss von Probanden

\begin{tabular}{|l|c|c|c|}
\hline \multirow{2}{*}{ Geschlecht } & Kontrollgruppe & Patientengruppe \\
\cline { 2 - 4 } & W & 8 & 12 \\
\hline Alter & MW & 7 & 5 \\
\hline Eingeschlossen & 17 & $51,82($ SD 7,282) \\
\hline Ausgeschlossen & 3 & 15 \\
\hline Gesamt & 20 & 17 \\
\hline
\end{tabular}

Tabelle 2: Suchtdruck (Craving) und Kontrolle vor und nach dem Experiment bei Kontrollen und Patienten. t-Test bei unabhängigen Stichproben

\begin{tabular}{|l|l|c|c|c|c|}
\hline \multicolumn{2}{|c|}{} & T & $\begin{array}{c}\text { Sig. } \\
\text { (2-seitig) }\end{array}$ & $\begin{array}{c}\text { MW } \\
\text { Patient }\end{array}$ & $\begin{array}{c}\text { MW } \\
\text { Kontrolle }\end{array}$ \\
\hline $\begin{array}{l}\text { Vor dem } \\
\text { Experiment }\end{array}$ & Suchtdruck (Craving) & 1,669 & 0,106 & 11,76 & 2,00 \\
\cline { 2 - 6 } & Kontrolle & $-1,145$ & 0,261 & 93,82 & 99,00 \\
\hline $\begin{array}{l}\text { Nach dem } \\
\text { Experiment }\end{array}$ & Suchtdruck (Craving) & 1,421 & 0,166 & 11,18 & 2,67 \\
\cline { 2 - 6 } & Kontrolle & $-1,266$ & 0,215 & 93,24 & 99,67 \\
\hline
\end{tabular}

Tabelle 3: Suchtdruck (Craving) und Kontrolle vor und nach dem Experiment, t-Test bei gepaarten Stichproben

\begin{tabular}{|l|l|c|c|c|}
\hline \multicolumn{2}{|c|}{} & T & $\begin{array}{c}\text { Sig. } \\
\text { (2-seitig) }\end{array}$ & MW \\
\hline $\begin{array}{l}\text { Vor und nach dem } \\
\text { Experiment }\end{array}$ & Suchtdruck (Craving) & 0,000 & 1,000 & 0,000 \\
\cline { 2 - 5 } & Kontrolle & 0,000 & 1,000 & 0,000 \\
\hline
\end{tabular}


Tabelle 4: Unterschiede im Trinkverhalten zwischen Patienten und Kontrollen bzw. Männern und Frauen. Nicht parametrischer Test für unabhängige Stichprobe auf Gruppenebene und für das Geschlecht

\begin{tabular}{|l|l|c|}
\hline \multirow{2}{*}{$\begin{array}{l}\text { Trinkverhalten } \\
\text { Patienten vs. Kontrollen }\end{array}$} & \multicolumn{1}{|c|}{ Test } & Sig. \\
\hline \multirow{2}{*}{$\begin{array}{l}\text { Trinkverhalten } \\
\text { Männlich vs. Weiblich }\end{array}$} & Kruskal-Wallis & 0,306 \\
\cline { 2 - 3 } & Median & 0,181 \\
\cline { 2 - 3 } & Kruskal-Wallis & 0,673 \\
\hline
\end{tabular}

Tabelle 5: Signifikante Differenzen der Antwortzeiten zwischen Patienten und Kontrollen während der Messung im MRT. Einfaktorielle ANOVA

\begin{tabular}{|l|c|c|c|c|}
\hline \multicolumn{1}{|c|}{ Bedingung } & Kontrollen & Patienten & F & Signifikanz \\
\hline & \multicolumn{2}{|c|}{ Mittlere Antwortzeit [ms] } & & P \\
\hline Alk_rej & 837,6 & 966,3 & 4,38 & 0,045 \\
\hline Reason & 733,2 & 841,5 & 5,38 & 0,027 \\
\hline Reward & 939,8 & 1040,8 & 4,4 & 0,045 \\
\hline Target & 709,8 & 798,5 & 4,99 & 0,033 \\
\hline Target_alk & 641,9 & 750,8 & 9,44 & 0,004 \\
\hline $\begin{array}{l}\text { MW aller } \\
\text { Antworten }\end{array}$ & 840,5 & 929,6 & 3,79 & 0,061 \\
\hline
\end{tabular}

Tabelle 6: Vergleich der angenommenen und nicht angenommenen konditionierten Stimuli unter den beiden Gruppen

\begin{tabular}{|l|l|c|c|}
\hline \multirow{2}{*}{ Reward_Anzahl } & Kontrolle & MW & Signifikanz \\
\cline { 2 - 4 } & Patient & 45,73 & 0,019 \\
\hline \multirow{2}{*}{ Reward_miss_Anzahl } & Kontrolle & 39,24 & \\
\cline { 2 - 4 } & Patient & 2,75 & 0,019 \\
\hline
\end{tabular}


Tabelle 7: Anzahl der angenommenen alkoholischen Stimuli im Vergleich zu den anderen Stimuli, in beiden Gruppen

\begin{tabular}{|l|c|c|c|c|c|}
\hline & Kontrollen & Patienten & F & Signifikanz & \\
\hline & \multicolumn{3}{|c|}{ Mittelwert angenommene Stimuli } & \\
\hline Alkohol & 7,1 & 21,1 & 5,42 & 0,038 & $\begin{array}{c}\mathrm{N}=7 \text { für jede } \\
\text { Gruppe, alle } \\
\text { anderen haben } \\
\text { keine Alk.-Bilder } \\
\text { angenommen }\end{array}$ \\
\hline
\end{tabular}

Tabelle 8: Replikation DRD

\begin{tabular}{|l|c|c|c|c|c|c|l|}
\hline Region & T-Wert & Koordinate & P Wert & $\begin{array}{c}\text { Svc } \\
\text { Koordinate }\end{array}$ & & \\
\hline Con2 & & & & & & & \\
\hline R VTA & 4,187 & {$[2 ;-14 ;-16]$} & 0,016 & 4 & -16 & -20 & Diekhof \& Gruber 2011 \\
\hline R NAC & 3,271 & {$[8 ; 14 ;-6]$} & 0,059 & 12 & 12 & -4 & Diekhof \& Gruber 2011 \\
\hline Con3 & & & & & & & \\
\hline L NAC & 4,440 & {$[-14 ; 12 ;-8]$} & 0,013 & 12 & 12 & -4 & Diekhof \& Gruber 2011 \\
\hline R NAC & 4,296 & {$[12 ; 8 ;-2]$} & 0,016 & 12 & 12 & -4 & Diekhof \& Gruber 2011 \\
\hline & & & & & & & \\
\hline R VTA & 3,036 & {$[4 ;-12 ;-18]$} & 0,090 & 4 & -16 & -20 & Diekhof \& Gruber 2011 \\
\hline
\end{tabular}




\subsection{Abbildungen}

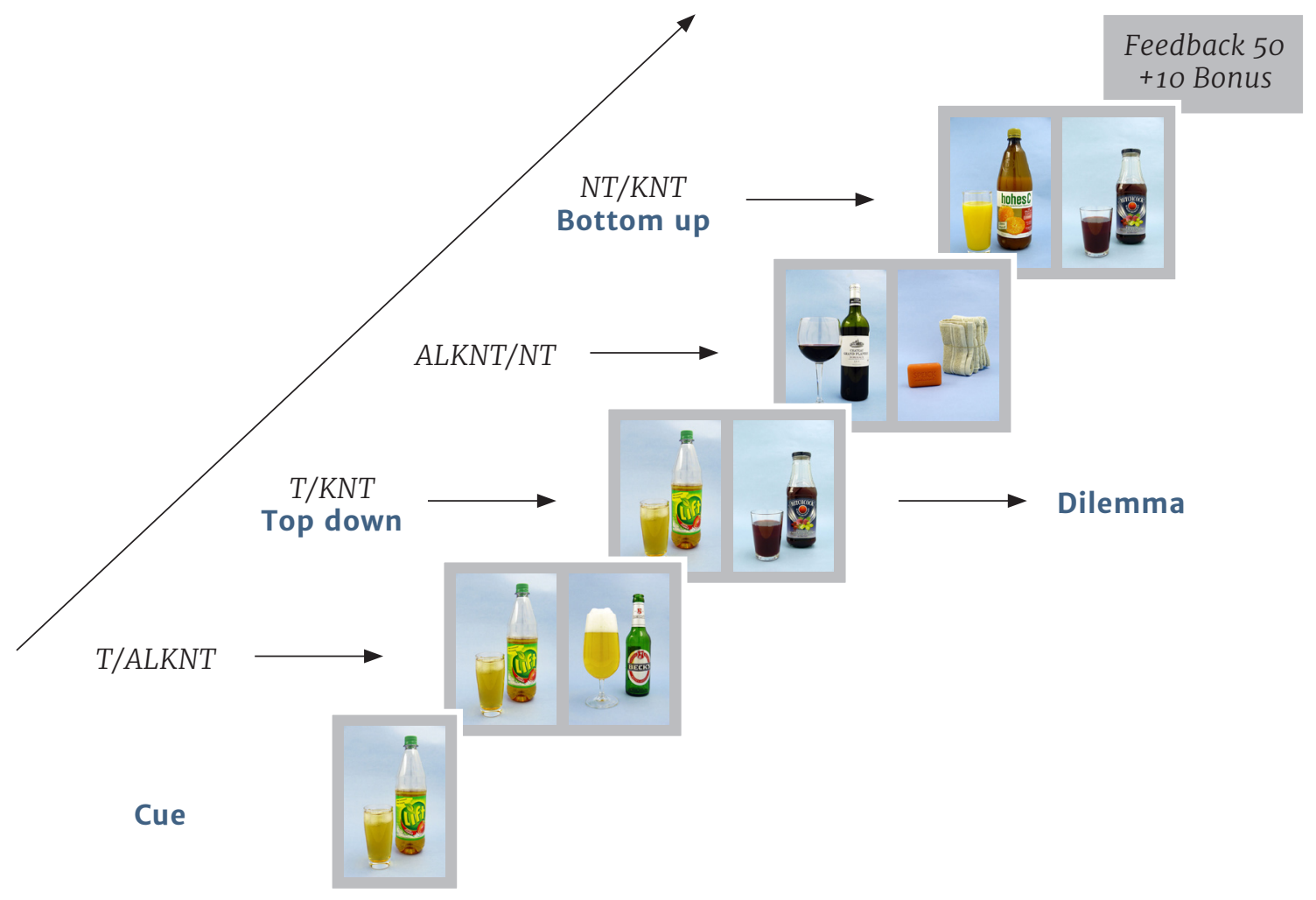

Abbildung 1: DRD-F1o-Paradigma: Die Probanden führten ein Entscheidungsfindungsparadigma aus, wobei sie sich zwischen unmittelbarer und langfristiger Belohnung entscheiden mussten. Das übergeordnete Ziel am Ende jedes erfolgreich bearbeiteten Blocks waren 50 Punkte. Am Anfang eines einzelnen Blocks wurde ein Hinweisreizbild (Cue) gezeigt, welches als Target (T) zielführend innerhalb jenes Blocks ausgewählt werden musste. Um zusätzliche 10 Bonuspunkte zu erhalten, konnten auch zuvor konditionierte Bilder (KNT) akzeptiert werden, wenn diese nicht parallel mit T präsentiert wurden (Dilemma). Eine fehlerhafte Annahme eines KNT bei paralleler Präsentation mit einem T führte zum Verlust von 50 Punkten. 


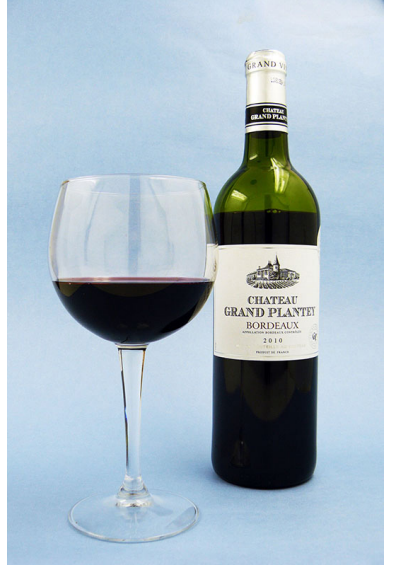

a) Alk

= alkoholisches Getränk

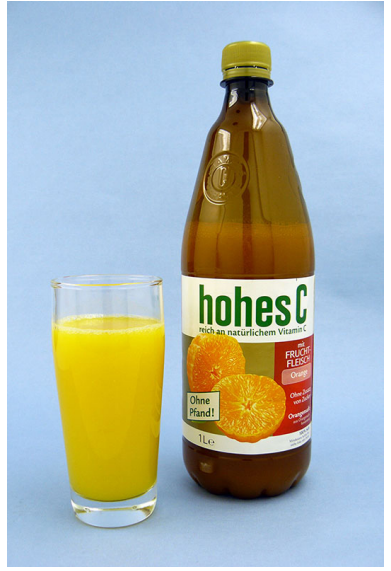

a) Non-alk

= nicht-alkoholisches Getränk

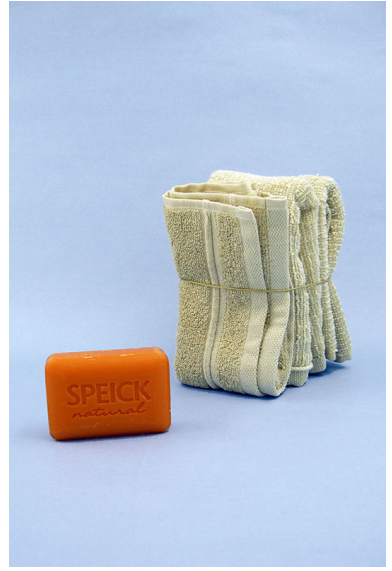

a) $\mathrm{OBJ}$

= neutrales Objekt

Abbildung 2: Beispielbilder 


\subsection{Stimulusmaterial}

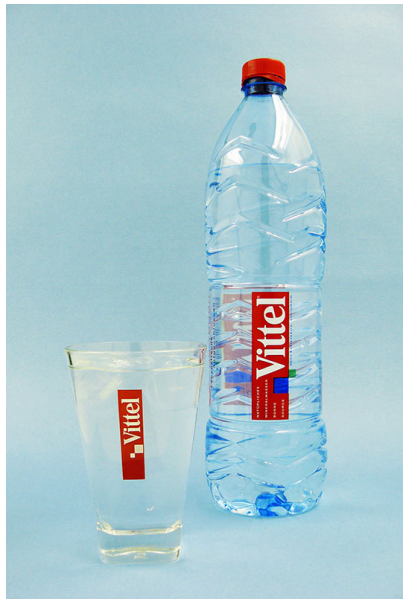

nalk2_vitt_label2.jpg

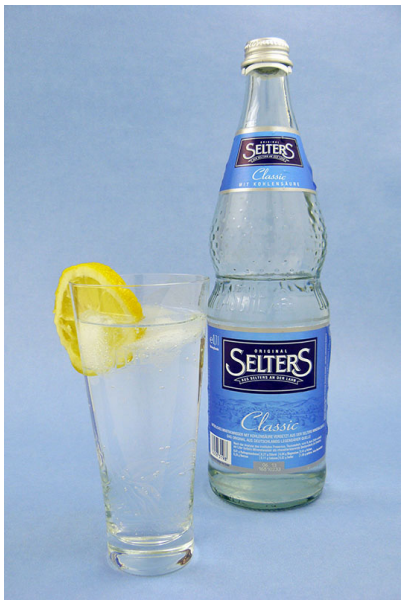

nalk2_selt_label1.jpg

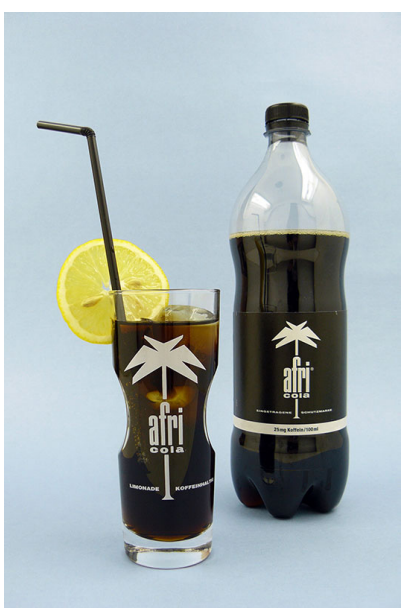

nalk2_afri_label2.jpg
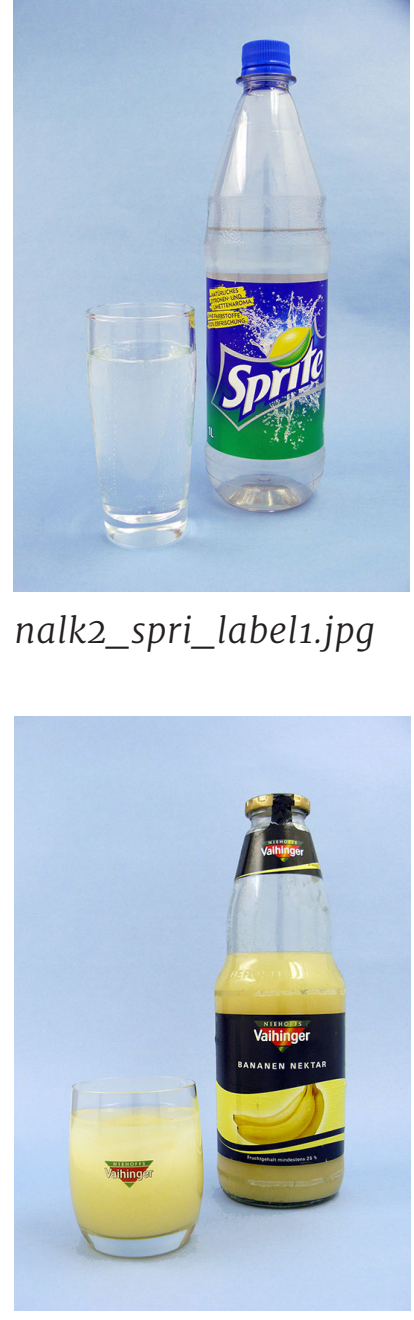

nalk2_vaih_label2.jpg

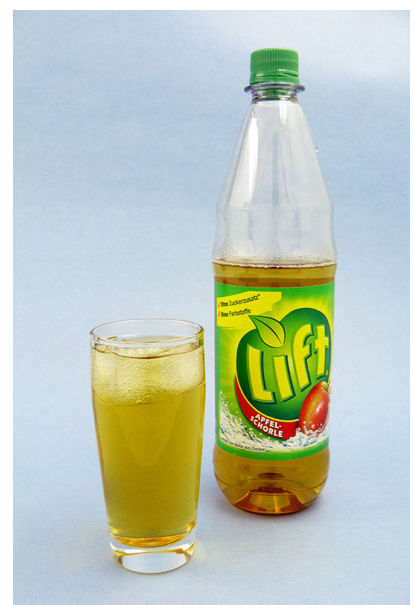

nalk2_lift_label1.jpg

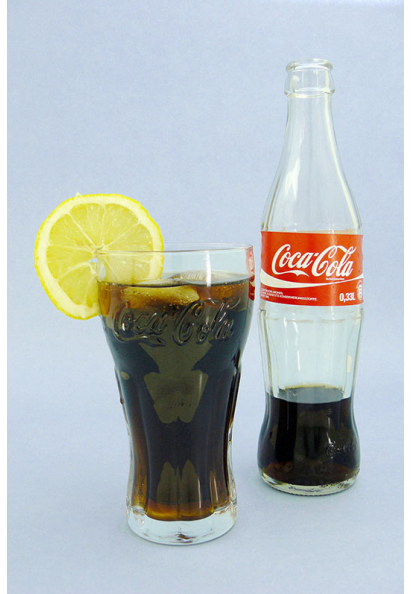

nalk2_coke_label2.jpg

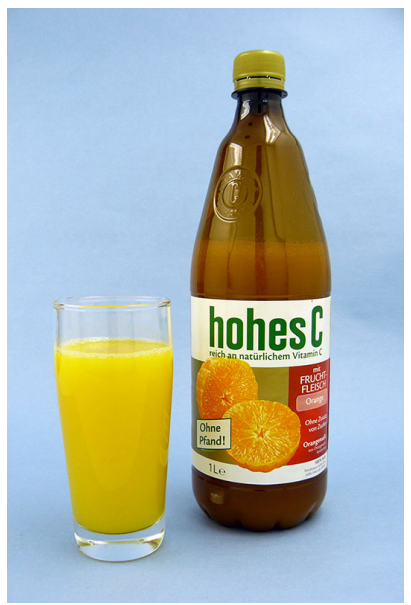

nalk2_hohC_label1.jpg

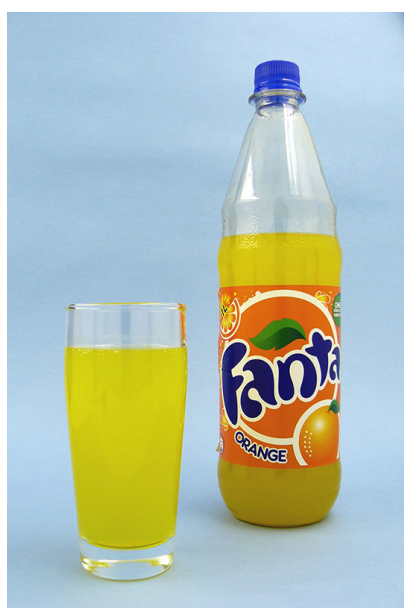

nalk2_fant_label1.jpg 


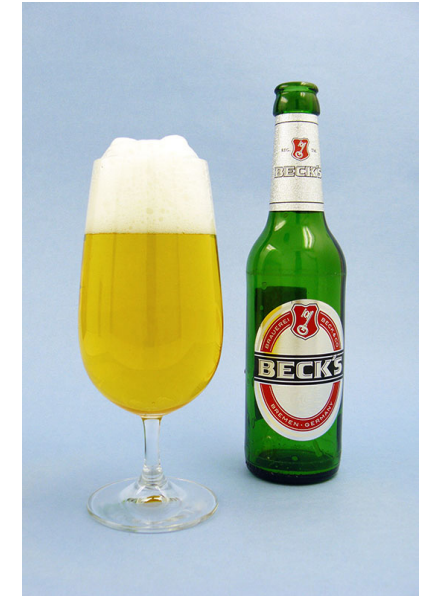

alk2_beck_label1.jpg

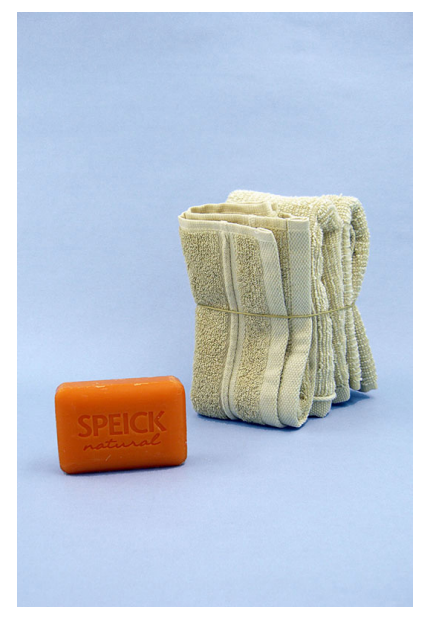

neut2_soap_label1.jpg

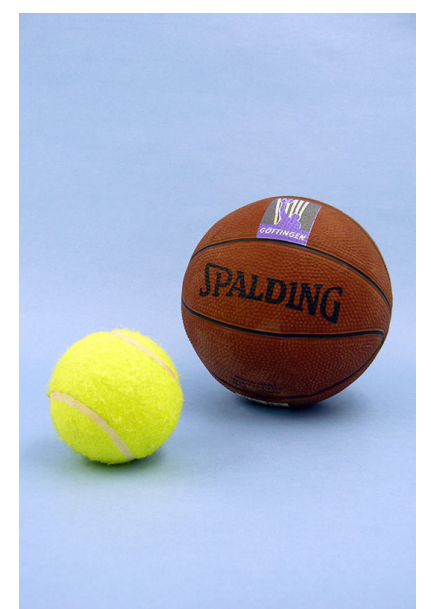

neut2_ball_label1.jpg

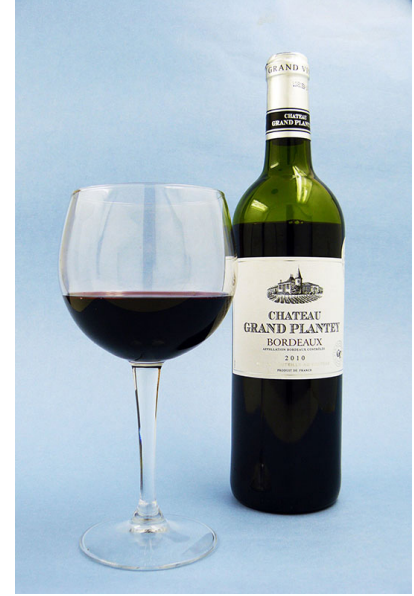

alk2_bord_label1.jpg

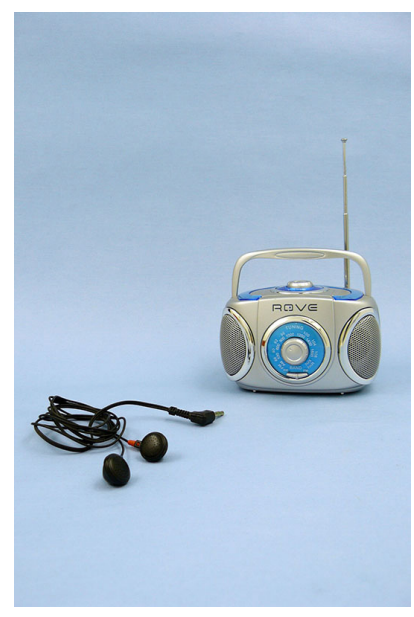

neut2_radio_label1.jpg

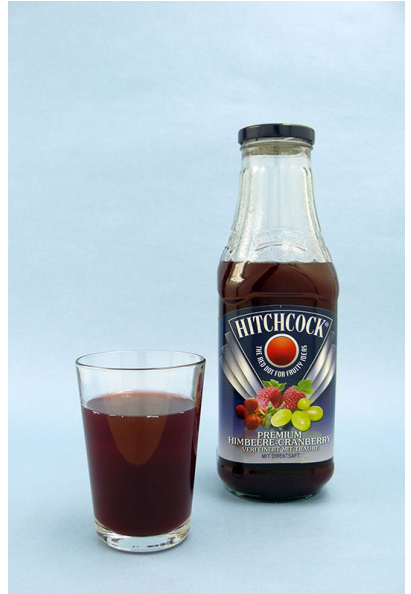

nalk2_hitch_label1.jpg

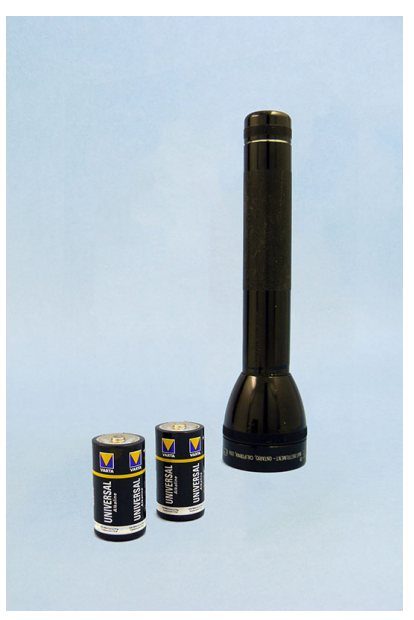

neut2_batt_label1.jpg 


\subsection{Fragebögen}

Visuelle Analog-Skala

Skala MR Messung

Datum:

Uhrzeit:

Probanden ID:

Name:

Vorname:

Wie groß ist jetzt Ihr Drang Alkohol zu trinken?

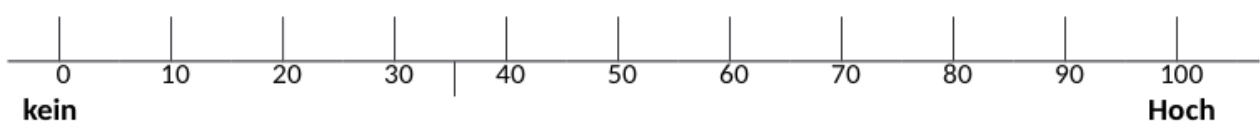

Wie stark können Sie diesen Drang kontrollieren?

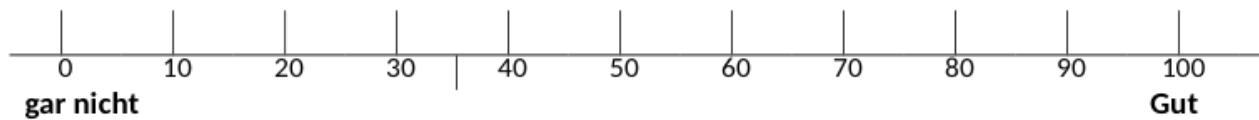


Bogen zum Trinkverhalten

(Patienten)

Wenn Sie in der Vergangenheit Alkohol getrunken haben, welche Getränke haben Sie dann wie häufig konsumiert?

Pils/Export
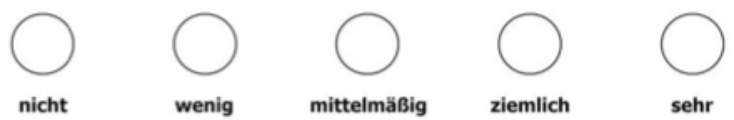

nicht

wenig

mittelmäßig

ziemlich

sehr

Alt-/Dunkelbier
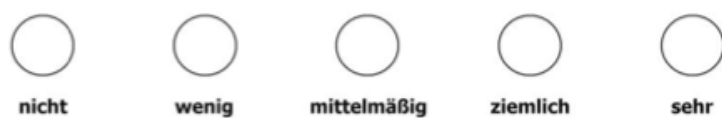

nicht

wenig

mittelmäßig

ziemlich

sehr

Weizen
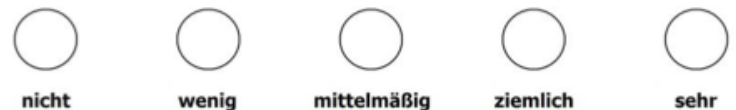

ziemlich

sehr

Weißwein
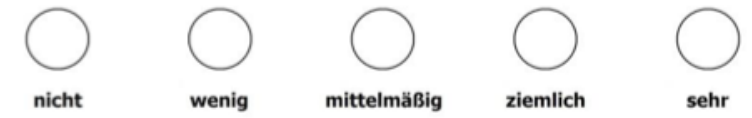


\section{Rotwein}
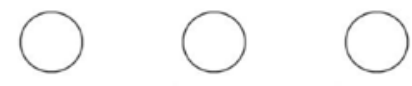

mittelmäßig
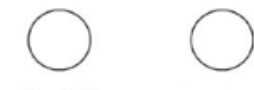

nicht

wenig

mittelmäBis

ziemlich

sehr

Sekt
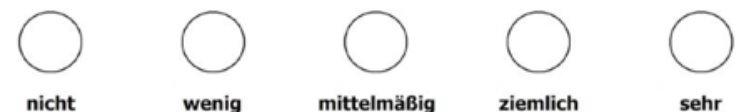

mittelmäßig

ziemlich

sehr

Schnaps/Korn
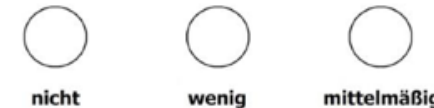

mittelmäßig
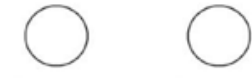

ziemlich

sehr

Whiskey/Cognac
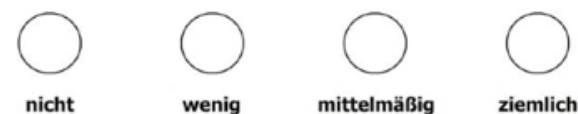

mittelmäßig ziemlich

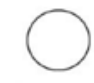


(Kontrollen)

Wenn Sie Alkohol trinken, wie gern bzw. häufig konsumieren Sie die folgenden Getränke?

Pils/Export
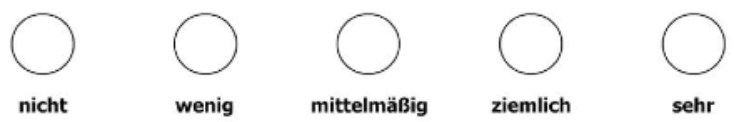

nicht

wenig

\section{mittelmasig}

ziemlich

sehr

Alt-/Dunkelbier
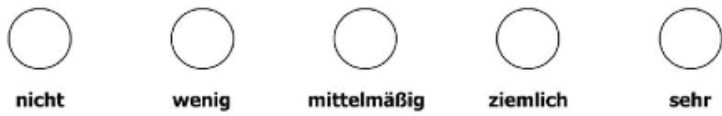

nicht

wenig

mittelmäßig

ziemlich

sehr

Weizen
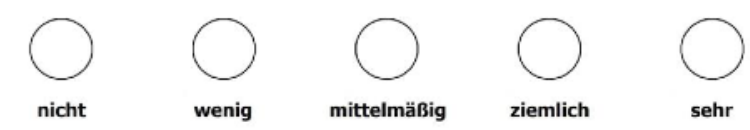

mittelmäßig

sehr

\section{Weißwein}
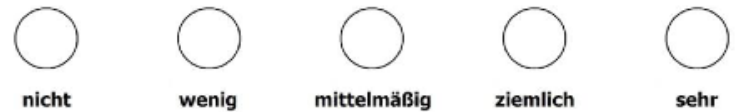

nicht

wenig

mittelmäßig

ziemlich

sehr 


\section{Rotwein}
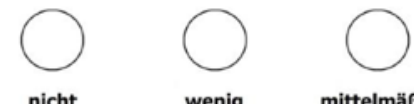

mittelmäßig
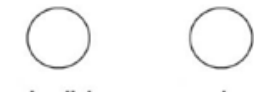

nicht

wenig

ziemlich

sehr

Sekt
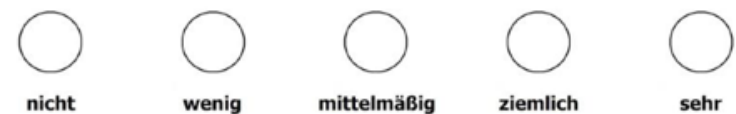

mittelmäßig

sehr

\section{Schnaps/Korn}
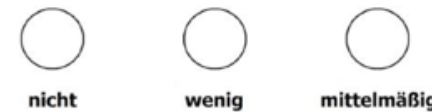

mittelmäßig
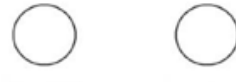

ziemlich

sehr

Whiskey/Cognac
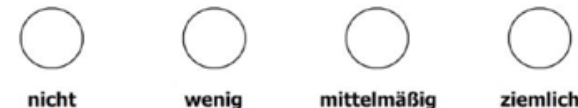

mittelmäßig

ziemlich

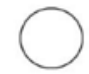




\subsection{Kontrastliste}

\section{Contrastliste}

\begin{tabular}{|c|c|c|c|c|c|c|c|c|c|c|}
\hline $\begin{array}{l}\text { Conl } \\
\text { D } \\
\end{array}$ & Contrast Name & type & PPI & NT_NT & NT_T & NT_KNT & NT_ALKNT & T_KNT & T_ALKNT OBJ & $\angle \mathrm{OBJ}$ \\
\hline 1 & Effect of Interest & $\mathrm{E}$ & 0 & 0 & 0 & 0 & 0 & 0 & 0 & 0 \\
\hline 2 & Reward, bottom up (Desire) & $\mathrm{T}$ & 0 & -1 & 0 & 1 & 0 & 0 & 0 & 0 \\
\hline 3 & Reason, top down supp & $\mathrm{T}$ & 0 & 0 & 0 & 1 & 0 & -1 & 0 & 0 \\
\hline 4 & Effect of Target on Reward (Reason) & $T$ & 0 & 0 & -1 & 0 & 0 & 1 & 0 & 0 \\
\hline 5 & Interaction Reward Reason Target (D-R) & $\mathrm{T}$ & 0 & -1 & 1 & 1 & 0 & -1 & 0 & 0 \\
\hline 6 & NT_NT_act & $\mathrm{T}$ & 0 & 1 & 0 & 0 & 0 & 0 & 0 & 0 \\
\hline 7 & NT_T_act & $T$ & 0 & 0 & 1 & 0 & 0 & 0 & 0 & 0 \\
\hline 8 & NT_KNT_act & $T$ & 0 & 0 & 0 & 1 & 0 & 0 & 0 & 0 \\
\hline 9 & NT_ALKNT_act & $T$ & 0 & 0 & 0 & 0 & 1 & 0 & 0 & 0 \\
\hline 10 & T_KNT_act & $T$ & 0 & 0 & 0 & 0 & 0 & 1 & 0 & 0 \\
\hline 11 & T_ALKNT_act & $T$ & 0 & 0 & 0 & 0 & 0 & 0 & 1 & 0 \\
\hline 12 & OBJ_OBJ_act & $T$ & 0 & 0 & 0 & 0 & 0 & 0 & 0 & 1 \\
\hline 13 & NT_NT_deact & $T$ & 0 & -1 & 0 & 0 & 0 & 0 & 0 & 0 \\
\hline 14 & NT_T_deact & $T$ & 0 & 0 & -1 & 0 & 0 & 0 & 0 & 0 \\
\hline 15 & NT_KNT_deact & $T$ & 0 & 0 & 0 & -1 & 0 & 0 & 0 & 0 \\
\hline 16 & NT_ALKNT_deact & $T$ & 0 & 0 & 0 & 0 & -1 & 0 & 0 & 0 \\
\hline 17 & T_KNT_deact & $T$ & 0 & 0 & 0 & 0 & 0 & -1 & 0 & 0 \\
\hline 18 & T_ALKNT_deact & $T$ & 0 & 0 & 0 & 0 & 0 & 0 & -1 & 0 \\
\hline 19 & OBJ_OBJ_deact & $T$ & 0 & 0 & 0 & 0 & 0 & 0 & 0 & -1 \\
\hline 20 & Alkohol bottom up (Desire) & $\mathrm{T}$ & 0 & -1 & 0 & 0 & 1 & 0 & 0 & 0 \\
\hline 21 & Reason, top down supp & $T$ & 0 & 0 & 0 & 0 & 1 & 0 & -1 & 0 \\
\hline 22 & Effect of Target on Alkohol (Reason) & $T$ & 0 & 0 & -1 & 0 & 0 & 0 & 1 & 0 \\
\hline 23 & Interaction Alkohol Reason Target (D-R) & $T$ & 0 & -1 & 1 & 0 & 1 & 0 & -1 & 0 \\
\hline
\end{tabular}




\section{LITERATURVERZEICHNIS}

Adams M, Effertz T: Volkswirtschaftliche Kosten des Alkohol- und Tabakkonsums. In: Singer MV, Batra A, Mann K (Hrsg.): Alkohol und Tabak. Grundlagen und Folgeerkrankungen. Thieme, Stutgart, New York 2011, 57-62

Adcock RA, Thangavel A, Whitfield-Gabrieli S, Knutson B, Gabrieli JDE (2006): Reward-motivated learning: mesolimbic activation precedes memory formation. Neuron 50, 507-517

American Psychiatric Association: Diagnostic and statistical manual of mental disorders: DSM-IV. 4. Auflage; Washington 1998

Bechara A, Damasio H, Damasio AR, Lee GP (1999): Different contributions of the human amygdala and ventromedial prefrontal cortex to decision-making. J Neurosci 19, 5473-5481

Beck A, Schlagenhauf F, Wüstenberg T, Hein J, Kienast T, Kahnt T, Schmack K, Hägele C, Knutson B, Heinz A, Wrase J (2009): Ventral striatal activation during reward anticipation correlates with impulsivity in alcoholics. Biol Psychiatry 66, 734-742

Bergmann E, Horch K: Kosten alkoholassoziierter Krankheiten: Schätzungen für Deutschland. Berlin 2002

Berridge KC (2007): The debate over dopamine's role in reward: the case for incentive salience. Psychopharmacology (Berl) 191, 391-431

Berridge KC, Robinson TE (2003): Parsing reward. Trends Neurosci 26, 507-513

Berridge KC, Robinson TE, Aldridge JW (2009): Dissecting components of reward: 'liking', 'wanting', and learning. Curr Opin Pharmacol 9, $65-73$

Brodie MS, Shefner SA, Dunwiddie TV (1990): Ethanol increases the firing rate of dopamine neurons of the rat ventral tegmental area in vitro. Brain Res 508, 65-69

Bühringer G (2000): Testing CBT mechanisms of action: humans behave in a more complex way than our treatment studies would predict! Addiction 95, 1715-1716 
Burger M, Mensink GBM, Bergmann E, Pietrzik K (2003): Characteristics associated with alcohol consumption in Germany. J Stud Alcohol 64, $262-269$

Cao Z, Bennett M, Orr C, Icke I, Banaschewski T, Barker GJ, Bokde ALW, Bromberg U, Büchel C, Quinlan EB, et al. (2019): Mapping adolescent reward anticipation, receipt, and prediction error during the monetary incentive delay task. Hum Brain Mapp 40, 262-283

Daw ND, Niv Y, Dayan P (2005): Uncertainty-based competition between prefrontal and dorsolateral striatal systems for behavioral control. Nat Neurosci 8, 1704-1711

Daw ND, O'Doherty JP, Dayan P, Seymour B, Dolan RJ (2006): Cortical substrates for exploratory decisions in humans. Nature 441, 876-879

DHS: Deutsche Hauptstelle für Suchtfragen e.V., Bundeszentrale für gesundheitliche Aufklärung (BzgA) (Hrsg.): Alkohol - Basisinformationen. 14. Auflage; DHS, Hamm 2017, http://www.dhs.de/fileadmin/user upload/pdf/Broschueren/2017 Basisinfo Alkohol.pdf

Diekhof EK, Gruber O (2010): When desire collides with reason: functional interactions between anteroventral prefrontal cortex and nucleus accumbens underlie the human ability to resist impulsive desires. $J$ Neurosci 30, 1488-1493

Diekhof EK, Falkai P, Gruber O (2008): Functional neuroimaging of reward processing and decision-making: a review of aberrant motivational and affective processing in addiction and mood disorders. Brain Res Rev 59, 164-184

Diekhof EK, Nerenberg L, Falkai P, Dechent P, Baudewig J, Gruber O (2012a): Impulsive personality and the ability to resist immediate reward: an fMRI study examining interindividual differences in the neural mechanisms underlying self-control. Hum Brain Mapp 33, 2768-2784

Diekhof EK, Kaps L, Falkai P, Gruber O (2012b): The role of the human ventral striatum and the medial orbitofrontal cortex in the representation of reward magnitude - an activation likelihood estimation meta-analysis of neuroimaging studies of passive reward expectancy and outcome processing. Neuropsychologia 50, 1252-1266 
DSM IV: Diagnostisches und statistisches Manual psychischer Störungen: Textrevision - DSM-IV-TR; übersetzt nach der Textrevision der 4. Auflage / Dt. Bearb. und Einführung von Saß H, Wittchen H-U, Zau$\operatorname{dig} M$, Houben I. Hogrefe, Verlag für Psychologie, Göttingen; Bern; Toronto; Seattle; 2003

Feuerlein W: Alkoholismus: Warnsignale, Vorbeugung, Therapie. 5. Auflage; C.H. Beck, München 2005

Gessa GL, Muntoni F, Collu M, Vargiu L, Mereu G (1985): Low doses of ethanol activate dopaminergic neurons in the ventral tegmental area. Brain Res 348, 201-203

Goldstein RZ, Volkow ND (2002): Drug addiction and its underlying neurobiological basis: neuroimaging evidence for the involvement of the frontal cortex. Am J Psychiatry 159, 1642-1652

Gruber O, Melcher T, Diekhof EK, Karch S, Falkai P, Goschke T (2009): Brain mechanisms associated with background monitoring of the environment for potentially significant sensory events. Brain Cogn 69, $559-564$

Haber SN, Knutson B (2010): The reward circuit: linking primate anatomy and human imaging. Neuropsychopharmacology 35, 4-26

Hare TA, Camerer CF, Rangel A (2009): Self-control in decision-making involves modulation of the VMPFC valuation system. Science 324, $646-648$

Havemann U, Kuschinsky K (1982): Neurochemical aspects of the opioid-induced „catatonia“. Neurochem Int 4, 199-215

Havemann U, Kuschinsky K (1985): Locomotor activity of rats after injection of various opioids into the nucleus accumbens and the septum mediale. Naunyn Schmiedebergs Arch Pharmacol 331, 175-180

Hebscher M, Barkan-Abramski M, Goldsmith M, Aharon-Peretz J, Gilboa A (2016): Memory, Decision-Making, and the Ventromedial Prefrontal Cortex (vmPFC): The Roles of Subcallosal and Posterior Orbitofrontal Cortices in Monitoring and Control Processes. Cereb Cortex 26, $4590-4601$ 
Heinz A, Siessmeier T, Wrase J, Hermann D, Klein S, Grüsser SM, Grüsser-Sinopoli SM, Flor H, Braus DF, Buchholz HG, et al. (2004): Correlation between dopamine $D(2)$ receptors in the ventral striatum and central processing of alcohol cues and craving. Am J Psychiatry $161,1783-1789$

Huskisson EC (1974): Measurement of pain. Lancet 2, 1127-1131

Kalenscher T, Ohmann T, Güntürkün O (2006): The neuroscience of impulsive and self-controlled decisions. Int J Psychophysiol 62, 203-211

Kalivas PW, Volkow ND (2005): The neural basis of addiction: a pathology of motivation and choice. Am J Psychiatry 162, 1403-1413

Kareken DA, Claus ED, Sabri M, Dzemidzic $M$, Kosobud AEK, Radnovich AJ, Hector D, Ramchandani VA, O'Connor SJ, Lowe M, Li T-K (2004): Alcohol-related olfactory cues activate the nucleus accumbens and ventral tegmental area in high-risk drinkers: preliminary findings. Alcohol Clin Exp Res 28, 550-557

Kim CK, Ye L, Jennings JH, Pichamoorthy N, Tang DD, Yoo A-CW, Ramakrishnan C, Deisseroth K (2017): Molecular and Circuit-Dynamical Identification of Top-Down Neural Mechanisms for Restraint of Reward Seeking. Cell 170, 1013-1027.e14

Knutson B, Fong GW, Adams CM, Varner JL, Hommer D (2001): Dissociation of reward anticipation and outcome with event-related fMRI. Neuroreport 12, 3683-3687

Koob GF: Allostatic view of motivation: implications for psychopathology. In: Bevins RA, Bardo MT: Motivational factors in the etiology of drug abuse. Nebraska Symposium on Motivation 50, University of Nebraska Press, Lincoln 2004, 1-18, http://www.nebraskapress.unl.edu/ university-of-nebraska-press/9780803213401

Koob GF (2008): A role for brain stress systems in addiction. Neuron 59, $11-34$

Koob GF (2013): Theoretical frameworks and mechanistic aspects of alcohol addiction: alcohol addiction as a reward deficit disorder. Curr Top Behav Neurosci 13, 3-30 
Koob GF, Le Moal M (1997): Drug abuse: hedonic homeostatic dysregulation. Science 278, 52-58

Koob GF, Volkow ND (2010): Neurocircuitry of addiction. Neuropsychopharmacology 35, 217-238

Logothetis NK (2008): What we can do and what we cannot do with fMRI. Nature 453, 869-878

Luijten M, Schellekens AF, Kühn S, Machielse MWJ, Sescousse G (2017): Disruption of Reward Processing in Addiction: An Image-Based Meta-analysis of Functional Magnetic Resonance Imaging Studies. JAMA Psychiatry 74, 387-398

Martin-Sölch C, Magyar S, Künig G, Missimer J, Schultz W, Leenders KL (2001): Changes in brain activation associated with reward processing in smokers and nonsmokers. A positron emission tomography study. Exp Brain Res 139, 278-286

Oades RD, Halliday GM (1987): Ventral tegmental (A10) system: neurobiology. 1. Anatomy and connectivity. Brain Res 434, 117-165

O'Doherty JP (2004): Reward representations and reward-related learning in the human brain: insights from neuroimaging. Curr Opin Neurobiol 14, 769-776

O'Doherty JP, Deichmann R, Critchley HD, Dolan RJ (2002): Neural responses during anticipation of a primary taste reward. Neuron 33, $815-826$

Ogawa S, Lee TM, Kay AR, Tank DW (199o): Brain magnetic resonance imaging with contrast dependent on blood oxygenation. Proc Natl Acad Sci USA 87, 9868-9872

Olds J, Milner P (1954): Positive reinforcement produced by electrical stimulation of septal area and other regions of rat brain. J Comp Physiol Psychol 47, 419-427

Robinson TE, Berridge KC (1993): The neural basis of drug craving: an incentive-sensitization theory of addiction. Brain Res Brain Res Rev $18,247-291$ 
Sauer A (2014): Erstellung und Validierung eines Bildinventars zur Untersuchung motivationaler Prozesse bei Alkoholpatienten. Unveröffentlicht, liegt der Verfasserin vor.

Schäfer M, Heinz A: Neurobiologie der Alkoholabhängigkeit. In: Singer M, Teyssen S (Hrsg.): Alkohol und Alkoholfolgekrankheiten; Springer, Berlin, Heidelberg 2005, 480-487

Schoenbaum G, Chiba AA, Gallagher M (1998): Orbitofrontal cortex and basolateral amygdala encode expected outcomes during learning. Nat Neurosci 1, 155-159

Tremblay L, Schultz W (1999): Relative reward preference in primate orbitofrontal cortex. Nature 398, 704-708

Volkow ND, Fowler JS (2000): Addiction, a disease of compulsion and drive: involvement of the orbitofrontal cortex. Cereb Cortex 10, 318-325

Volkow ND, Fowler JS, Wang G-J (2002): Role of dopamine in drug reinforcement and addiction in humans: results from imaging studies. Behav Pharmacol 13, 355-366

Volkow ND, Wang G-J, Telang F, Fowler JS, Logan J, Jayne M, Ma Y, Pradhan $K$, Wong $C$ (2007): Profound decreases in dopamine release in striatum in detoxified alcoholics: possible orbitofrontal involvement. J Neurosci 27, 12700-12706

Walter H, Abler B, Ciaramidaro A, Erk S (2005): Motivating forces of human actions. Neuroimaging reward and social interaction. Brain Res Bull $67,368-381$

Wedekind D, Herchenhein T, Kirchhainer J, Bandelow B, Falkai P, Engel K, Malchow B, Havemann-Reinecke U (2010): Serotonergic function, substance craving, and psychopathology in detoxified alcohol-addicted males undergoing tryptophan depletion. J Psychiatr Res 44, 1163-1169

WHO: Internationale Klassifikation psychischer Störungen: ICD-10, Kapitel $V(F)$; klinisch-diagnostische Leitlinien. Hrsg. v. Dilling H, Mombour W, Schmidt MH. 6. Auflage; Huber, Bern 2008

Williams LM (2006): An integrative neuroscience model of "significance“ processing. J Integr Neurosci 5, 1-47 
Worsley KJ, Marrett S, Neelin P, Vandal AC, Friston KJ, Evans AC (1996): A unified statistical approach for determining significant signals in images of cerebral activation. Hum Brain Mapp 4, 58-73

Wrase J, Grüsser SM, Klein S, Diener C, Hermann D, Flor H, Mann K, Braus DF, Heinz A (2002): Development of alcohol-associated cues and cue-induced brain activation in alcoholics. Eur Psychiatry 17, 287291

Wrase J, Schlagenhauf F, Kienast T, Wüstenberg T, Bermpohl F, Kahnt T, Beck A, Ströhle A, Juckel G, Knutson B, Heinz A (2007): Dysfunction of reward processing correlates with alcohol craving in detoxified alcoholics. Neuroimage 35, 787-794

Wrase J, Makris N, Braus DF, Mann K, Smolka MN, Kennedy DN, Caviness VS, Hodge SM, Tang L, Albaugh M, et al. (2008): Amygdala volume associated with alcohol abuse relapse and craving. Am J Psychiatry $165,1179-1184$

Yacubian J, Sommer T, Schroeder K, Gläscher J, Braus DF, Büchel C (2007): Subregions of the ventral striatum show preferential coding of reward magnitude and probability. Neuroimage 38, 557-563

Zijlstra F, Veltman DJ, Booij J, van den Brink W, Franken IHA (20o9): Neurobiological substrates of cue-elicited craving and anhedonia in recently abstinent opioid-dependent males. Drug Alcohol Depend 99, 183-192 


\section{Danksagung}

Ich bedanke mich an erster Stelle bei Herrn Prof. Dr. med. Oliver Gruber, der es mir durch die Breitstellung dieses Themas und die Aufgabenstellung ermöglicht hat, diese Promotion durchzuführen.

Mein ganz herzlicher Dank gilt Dr. Bernd Krämer. Danke für die großartige Betreuung und Unterstützung, für die Beantwortung all meiner Fragen und für die unglaubliche Geduld in den letzten Monaten.

Andrea Sauer danke ich besonders für die Bereitstellung des Stimulusmaterials.

Britta Perl und Ilona Pfalert danke ich für die Unterstützung und die tolle Zeit im fMRT-Labor.

Ich danke weiter Dr. Tobias Melcher, Dr. Henning Vieker, Eiko Lasjcsak und Maria Keil für die Unterstützung.

Außerdem möchte ich mich ganz herzlich bei allen Probanden für die Teilnahme an der Studie bedanken. 